\title{
A variant of Horn's problem and the derivative principle
}

\author{
Lin Zhang ${ }^{1,2 *}$ Hua Xiang ${ }^{3 \dagger}$ \\ ${ }^{1}$ Institute of Mathematics, Hangzhou Dianzi University, Hangzhou 310018, PR China \\ ${ }^{2}$ Max-Planck-Institute for Mathematics in the Sciences, Leipzig 04103, Germany \\ ${ }^{3}$ School of Mathematics and Statistics, Wuhan University, Wuhan 430072, PR China
}

\begin{abstract}
Identifying the spectrum of the sum of two given Hermitian matrices with fixed eigenvalues is the famous Horn's problem. In this note, we investigate a variant of Horn's problem, i.e., we identify the probability density function (abbr. pdf) of the diagonals of the sum of two random Hermitian matrices with given spectra. We then use it to re-derive the pdf of the eigenvalues of the sum of two random Hermitian matrices with given eigenvalues via the derivative principle, a powerful tool used to get the exact probability distribution by reducing to the corresponding distribution of diagonal entries. We can also recover Jean-Bernard Zuber's recent results. Moreover, as an illustration, we derive the analytical expressions of eigenvalues of the sum of two random Hermitian matrices from $\operatorname{GUE}(n)$ or Wishart ensemble by the derivative principle, respectively. We also investigate the statistics of exponential of random matrices and connect them with Golden-Thompson inequality, and partially answer a question proposed by Forrester. Some potential applications in quantum information theory, such as uniform average quantum Jensen-Shannon divergence and average coherence of uniform mixture of two orbits, are discussed.
\end{abstract}

Mathematics Subject Classification. 22E70, 81Q10, 46L30, 15A90, 81R05

Keywords. Horn's problem; derivative principle; probability density function; quantum Jensen-Shannon divergence; quantum coherence

\section{Introduction}

The famous Horn's problem asks for the spectrum of the sum of two given Hermitian matrices with fixed eigenvalues. Specifically, Horn's problem characterizes the triple $(\boldsymbol{a}, \boldsymbol{b}, \boldsymbol{c})$ for

\footnotetext{
*E-mail: godyalin@163.com; linzhang@mis.mpg.de

†E-mail: hxiang@whu.edu.cn
} 
which there exist Hermitian matrices $\boldsymbol{A}, \boldsymbol{B}, \boldsymbol{C}$ with respective eigenvalues $\boldsymbol{a}=\left(a_{1}, \ldots, a_{n}\right), \boldsymbol{b}=$ $\left(b_{1}, \ldots, b_{n}\right)$ and $c=\left(c_{1}, \ldots, c_{n}\right)$ satisfying the following constraint:

$$
A+B=C
$$

This problem has an affirmative answer [5], in terms of linear inequalities which are now called Horn's inequalities. The solutions form a convex polytope whose describing inequalities have been conjectured by Horn in 1962 [12]. Note that the convex polytope for the solution of Horn's problem is, in general, nontrivial. Hence each point in this convex polytope corresponds to a possible eigenvalue vector for the sum, except the trivial cases (for example, when one of the matrices is scalar).

Although Horn's problem is apparently an elementary problem (a complete answer to Horn's problem takes almost a century), it turns out to be connected with many areas of mathematics: linear algebra of course [15], but also combinatorics, algebraic geometry [15], symplectic geometry, and even probability theory, etc. For instance, Alekseev et. al give a symplectic proof of the Horn inequalities on eigenvalues of a sum of two Hermitian matrices with given spectra [1], and Zuber investigate the probability distribution over the Horn's polytope [22]. Related probabilistic works include $[7,8]$.

Besides, some researchers give a description of the Duistermaat-Heckman measure on the Horn polytope. Mathematical speaking, the eigenvalue distributions involved are so-called Duistermaat-Heckman measures [6], which are defined using the push-forward of the Liouville measure on a symplectic manifold along the moment map. We follow the notations along the paper [6], and consider the problem of describing the sum of two coadjoint orbits $\mathcal{O}_{a}+\mathcal{O}_{b}$, where $K$ acts on $\mathcal{O}_{a} \times \mathcal{O}_{b}$ diagonally with moment map $(\boldsymbol{A}, \boldsymbol{B}) \mapsto \boldsymbol{A}+\boldsymbol{B}$ and we have [6]: Let $\boldsymbol{a} \in \mathfrak{t}_{>0}^{*}$ and $\boldsymbol{b} \in \mathfrak{t}_{\geqslant 0}^{*}$. Then $\mathrm{DH}_{\mathcal{O}_{a} \times \mathcal{O}_{b}}^{K}=\sum_{w \in W}(-1)^{l(w)} \delta_{w a} * \mathrm{DH}_{\mathcal{O}_{b}}^{T}$, where $l(w)$ is the length of the Weyl group element $w$; and DH is the Duistermaat-Heckamn measure. Apparently, this result gives the distribution of solutions of Horn's problem in theoretical level completely, but however in specific computational problems, it is less useful. In this paper, instead of Horn's polytope itself, we will consider the pdf of the diagonals of sum of two Hermitian matrices with prescribed spectra. Then by employing derivative principle, we obtain the probability distribution density of the eigenvalues of sum of two Hermitian matrices with prescribed spectra. The support for such probability distribution density function is just Horn's polytope, determined by Horn's inequalities. The obtained pdfs for the diagonals and spectra, respectively, are expressed by complicated complex integrals that can be explicitly calculated in lower dimensional cases, in particular for $2 \times 2$ case. We also apply this special case to analyze some quantities used in quantum information theory. We remark that Horn's problem is subsumed into the one-body quantum marginal problem, i.e., the problem of determining the set of possible reduced density 
matrices, known as the quantum marginal problem in quantum information theory and as the $N$-representability problem in quantum chemistry.

The paper is organized as follows. In Sect. 2, we derive an analytical formula for the pdf of the diagonals of sum of two random Hermitian matrices with prescribed spectra. The main results are summarized into Theorem 2.1 and Corollary 2.3. In Sect. 3, we firstly recall the derivative principle, and then combine this with the pdf of diagonals to derive the pdf of eigenvalues of sum of two random Hermitian matrices (see Theorem 3.2). We also find that the results obtained in [22] can be naturally derived from our main result. These materials are summarized into the Appendix A. We further derive the analytical expressions for eigenvalues of the sum of two random Hermitian matrices from $\operatorname{GUE}(n)$ or Wishart ensemble by derivative principle, respectively, which are summarized in Propositions 3.4. Sequentially, in Sect. 3.3, we use the pdf of the sum of two GUE random matrices to derive the expectation of the matrix exponential and partly answer a question proposed by Forrester [9]. As another application of our results, in Sect. 4, we use our lower dimensional result to analyze some quantities used in quantum information theory. Finally, we conclude with some remarks in Sect. 5.

\section{The pdf of diagonals of the sum of two random Hermitian matrices with given spectra}

We present an explicit formula concerning the joint distribution density of the diagonals of the sum of two random Hermitian matrices with given spectra. This is a new result in this note, and it will be used to derive the pdf of eigenvalues of the sum of two random Hermitian matrices with given spectra. To keep accordance with the notation in the present literature, the notation adopted here is only a little different from that in [18].

Denote $x=\left(x_{1}, \ldots, x_{n}\right), \widehat{x}=\operatorname{diag}\left(x_{1}, \ldots, x_{n}\right)$ and $\Delta(x)=\prod_{1 \leqslant i<j \leqslant n}\left(x_{i}-x_{j}\right),[\mathrm{d} x]=\prod_{j=1}^{n} \mathrm{~d} x_{j}$. We also denote the unitary orbit with spectrum $x$ by $\mathcal{U}(\boldsymbol{x})=\left\{\boldsymbol{U} \widehat{x} \boldsymbol{U}^{+}: \boldsymbol{U} \in \mathrm{U}(n)\right\}$, where $\mathrm{U}(n)$ stands for the $n \times n$ unitary matrix group.

Theorem 2.1. Assume that two random matrices $\boldsymbol{A}$ and $\boldsymbol{B}$ chosen uniformly on the unitary orbits $\mathcal{U}(\boldsymbol{a})$ and $\mathcal{U}(\boldsymbol{b})$, respectively, then the joint $p d f q\left(\boldsymbol{C}^{\text {diag }} \mid \boldsymbol{a}, \boldsymbol{b}\right)$ of the diagonal part $\boldsymbol{C}^{\text {diag }}$ of the sum $\boldsymbol{C}=\boldsymbol{A}+\boldsymbol{B}$ is given by the following integral

$$
q\left(\boldsymbol{C}^{\mathrm{diag}} \mid \boldsymbol{a}, \boldsymbol{b}\right)=\frac{M}{\Delta(\boldsymbol{a}) \Delta(\boldsymbol{b})} \int_{\mathbb{R}^{n}}[\mathrm{~d} \boldsymbol{x}] \frac{\operatorname{det}\left(e^{\mathrm{i} x_{i} a_{j}}\right) \operatorname{det}\left(e^{\mathrm{i} x_{i} b_{j}}\right)}{\Delta(\boldsymbol{x})^{2} \prod_{i=1}^{n} e^{\mathrm{i} x_{i} C_{i i}}},
$$

where

$$
M=\frac{\left(\prod_{k=1}^{n} \Gamma(k)\right)^{2}}{(2 \pi)^{n} \mathbf{i}^{n(n-1)}} .
$$


Proof. From [22], we see that the pdf of $C=U \widehat{\boldsymbol{a}} \boldsymbol{U}^{+}+\boldsymbol{V} \widehat{\boldsymbol{b}} \boldsymbol{V}^{\dagger}$ is given by ${ }^{1}$

$$
p(\boldsymbol{C} \mid \boldsymbol{a}, \boldsymbol{b})=\frac{1}{2^{n} \pi^{n^{2}}} \int[\mathrm{d} \boldsymbol{X}] e^{-\mathrm{i} \operatorname{Tr}(\boldsymbol{X C})} \int_{\mathrm{U}(n)} \int_{\mathrm{U}(n)} \mathrm{d} \mu_{\text {Haar }}(\boldsymbol{U}) \mathrm{d} \mu_{\text {Haar }}(\boldsymbol{V}) e^{\mathrm{i} \operatorname{Tr}\left(\boldsymbol{X} \boldsymbol{U} \widehat{a} \boldsymbol{U}^{+}\right)} e^{\mathrm{i} \operatorname{Tr}\left(\boldsymbol{X} \boldsymbol{V} \widehat{\boldsymbol{b}} \boldsymbol{V}^{+}\right)} .
$$

We will consider the following question, i.e., the probability density function of diagonals of sum of two Hermitian matrices:

$$
q\left(\boldsymbol{C}^{\mathrm{diag}} \mid \boldsymbol{a}, \boldsymbol{b}\right)=\int\left[\mathrm{d} \boldsymbol{C}^{\mathrm{off}}\right] p(\boldsymbol{C} \mid \boldsymbol{a}, \boldsymbol{b}) .
$$

Employ the following identity: $\boldsymbol{X}=\boldsymbol{X}^{\text {diag }} \oplus \boldsymbol{X}^{\text {off }}, \boldsymbol{C}=\boldsymbol{C}^{\text {diag }} \oplus \boldsymbol{C}^{\text {off }}$, and

$$
\delta\left(\boldsymbol{X}^{\mathrm{off}}\right)=\frac{1}{\pi^{n(n-1)}} \int\left[\mathrm{d} \boldsymbol{C}^{\mathrm{off}}\right] e^{-\mathrm{i} \operatorname{Tr}\left(\boldsymbol{X}^{\text {off }} C^{\text {off }}\right)} .
$$

It follows that

$$
\begin{aligned}
& q\left(\boldsymbol{C}^{\text {diag }} \mid \boldsymbol{a}, \boldsymbol{b}\right)=\int\left[\mathrm{d} \boldsymbol{C}^{\text {off }}\right] p(\boldsymbol{C} \mid \boldsymbol{a}, \boldsymbol{b}) \\
& =\frac{1}{2^{n} \pi^{n^{2}}} \int[\mathrm{d} \boldsymbol{X}] \int\left[\mathrm{d} \boldsymbol{C}^{\text {off }}\right] e^{-\mathrm{i} \operatorname{Tr}(\boldsymbol{X} \boldsymbol{C})} \int_{\mathrm{U}(n)} \int_{\mathrm{U}(n)} \mathrm{d} \mu_{\text {Haar }}(\boldsymbol{U}) \mathrm{d} \mu_{\text {Haar }}(\boldsymbol{V}) e^{\mathrm{i} \operatorname{Tr}\left(\boldsymbol{X} \boldsymbol{U} \widehat{a} \boldsymbol{U}^{+}\right)} e^{\mathrm{i} \operatorname{Tr}\left(\boldsymbol{X} \boldsymbol{V} \widehat{b} V^{+}\right)} \\
& =\frac{1}{2^{n} \pi^{n^{2}}} \int[\mathrm{d} \boldsymbol{X}] e^{-\mathrm{i} \operatorname{Tr}\left(\boldsymbol{X}^{\text {diag }} \boldsymbol{C}^{\text {diag }}\right)} \int\left[\mathrm{d} \boldsymbol{C}^{\text {off }}\right] e^{-\mathrm{i} \operatorname{Tr}\left(\boldsymbol{X}^{\text {off }} \boldsymbol{C}^{\text {off }}\right)} \\
& \quad \times \int_{\mathrm{U}(n)} \int_{\mathrm{U}(n)} \mathrm{d} \mu_{\text {Haar }}(\boldsymbol{U}) \mathrm{d} \mu_{\text {Haar }}(\boldsymbol{V}) e^{\mathrm{i} \operatorname{Tr}\left(\boldsymbol{X} \boldsymbol{U} \widehat{a} \boldsymbol{U}^{+}\right)} e^{\mathrm{i} \operatorname{Tr}\left(\boldsymbol{X} \boldsymbol{V} \widehat{b} \boldsymbol{V}^{+}\right)} .
\end{aligned}
$$

Thus,

$$
\begin{aligned}
q\left(\boldsymbol{C}^{\text {diag }} \mid \mathbf{a}, \mathbf{b}\right)= & \frac{1}{(2 \pi)^{n}} \int[\mathrm{d} \boldsymbol{X}] e^{-\mathrm{i} \operatorname{Tr}\left(\boldsymbol{X}^{\text {diag }} \boldsymbol{C}^{\text {diag }}\right)} \delta\left(\boldsymbol{X}^{\text {off }}\right) \\
& \times \int_{\mathrm{U}(n)} \int_{\mathrm{U}(n)} \mathrm{d} \mu_{\text {Haar }}(\boldsymbol{U}) \mathrm{d} \mu_{\text {Haar }}(\boldsymbol{V}) e^{\mathrm{i} \operatorname{Tr}\left(\boldsymbol{X} \boldsymbol{U} \widehat{a} \boldsymbol{U}^{+}\right)} e^{\mathrm{i} \operatorname{Tr}\left(\boldsymbol{X} \boldsymbol{V} \widehat{b} \boldsymbol{V}^{+}\right)} \\
= & \frac{1}{(2 \pi)^{n}} \int\left[\mathrm{d} \boldsymbol{X}^{\text {diag }}\right] e^{-\mathrm{i} \operatorname{Tr}\left(\boldsymbol{X}^{\text {diag }} \boldsymbol{C}^{\text {diag }}\right)} \int_{\mathrm{U}(n)} \int_{\mathrm{U}(n)} \mathrm{d} \mu_{\text {Haar }}(\boldsymbol{U}) \mathrm{d} \mu_{\text {Haar }}(\boldsymbol{V}) \\
& \times \int\left[\mathrm{d} \boldsymbol{X}^{\text {off }}\right] \delta\left(\boldsymbol{X}^{\text {off }}\right) e^{\mathrm{i} \operatorname{Tr}\left(\boldsymbol{X} \boldsymbol{U} \widehat{a} \boldsymbol{U}^{+}\right)} e^{\mathrm{i} \operatorname{Tr}\left(\boldsymbol{X} \boldsymbol{V} \widehat{b} \boldsymbol{V}^{+}\right)} .
\end{aligned}
$$

Therefore,

$$
\begin{aligned}
q\left(\boldsymbol{C}^{\text {diag }} \mid \boldsymbol{a}, \boldsymbol{b}\right)= & \frac{1}{(2 \pi)^{n}} \int\left[\mathrm{d} \boldsymbol{X}^{\text {diag }}\right] e^{-\mathrm{i} \operatorname{Tr}\left(\boldsymbol{X}^{\text {diag }} \boldsymbol{C}^{\text {diag }}\right)} \\
& \times \int_{\mathrm{U}(n)} \int_{\mathrm{U}(n)} \mathrm{d} \mu_{\text {Haar }}(\boldsymbol{U}) \mathrm{d} \mu_{\text {Haar }}(\boldsymbol{V}) e^{\mathrm{i} \operatorname{Tr}\left(\boldsymbol{X}^{\text {diag }} \boldsymbol{U} \widehat{a} \boldsymbol{U}^{+}\right)} e^{\mathrm{i} \operatorname{Tr}\left(\boldsymbol{X}^{\text {diag }} \widehat{\boldsymbol{b}} \boldsymbol{V}^{+}\right)}
\end{aligned}
$$

and

$$
\begin{aligned}
q\left(\boldsymbol{C}^{\mathrm{diag}} \mid \boldsymbol{a}, \boldsymbol{b}\right) & =\frac{1}{(2 \pi)^{n}} \int\left[\mathrm{d} \boldsymbol{X}^{\mathrm{diag}}\right] e^{-\mathrm{i} \operatorname{Tr}\left(\boldsymbol{X}^{\text {diag }} \boldsymbol{C}^{\text {diag }}\right)}\left(\prod_{k=1}^{n} \Gamma(k) \frac{\operatorname{det}\left(e^{\mathrm{i} \boldsymbol{X}_{i i} a_{j}}\right)}{\Delta\left(\mathrm{i} \boldsymbol{X}^{\mathrm{diag}}\right) \Delta(\boldsymbol{a})}\right)\left(\prod_{k=1}^{n} \Gamma(k) \frac{\operatorname{det}\left(e^{\mathrm{i} \boldsymbol{X}_{i i} b_{j}}\right)}{\Delta\left(\mathrm{i} \boldsymbol{X}^{\text {diag }}\right) \Delta(\boldsymbol{b})}\right) \\
& =\frac{1}{(2 \pi)^{n} \mathrm{i}^{2\left(\begin{array}{c}
n \\
2
\end{array}\right)}} \frac{\left(\prod_{k=1}^{n} \Gamma(k)\right)^{2}}{\Delta(\boldsymbol{a}) \Delta(\boldsymbol{b})} \int \frac{\left[\mathrm{d} \boldsymbol{X}^{\mathrm{diag}}\right]}{\Delta\left(\boldsymbol{X}^{\text {diag }}\right)^{2}} e^{-\mathrm{i} \operatorname{Tr}\left(\boldsymbol{X}^{\mathrm{diag}} \boldsymbol{C}^{\text {diag }}\right)} \operatorname{det}\left(e^{\mathrm{i} \boldsymbol{X}_{i i} a_{j}}\right) \operatorname{det}\left(e^{\mathrm{i} \boldsymbol{X}_{i i} b_{j}}\right) .
\end{aligned}
$$

\footnotetext{
${ }^{1}$ Note that the factor $2^{-n} \pi^{-n^{2}}$ is different from that used by Zuber, i.e., $(2 \pi)^{-n^{2}}$. There exists a symmetry condition about $X$, that is, Hermiticity of $X$, and $\delta(X)=2^{-n} \pi^{-n^{2}} \int \exp (\mathrm{i} \operatorname{Tr}(T X))[\mathrm{d} T]$.
} 
This completes the proof.

Lemma 2.2. For given real numbers $x_{j}$ and $\lambda_{j}$ where $j=1, \ldots, n$, denote $\bar{x}=\frac{1}{n} \sum_{k=1}^{n} x_{k}$, then have the following identities:

$$
\operatorname{det}\left(e^{\mathrm{i} x_{i} \lambda_{j}}\right)=e^{\mathrm{i} \bar{x} \sum_{k=1}^{n} \lambda_{k}} \sum_{\sigma \in S_{n}} \operatorname{sign}(\sigma) \prod_{k=1}^{n-1} \exp \left[\mathrm{i}\left(x_{k}-x_{k+1}\right)\left(\sum_{j=1}^{k} \lambda_{\sigma(j)}-\frac{k}{n} \sum_{j=1}^{n} \lambda_{j}\right)\right]
$$

and

$$
\prod_{k=1}^{n} e^{-\mathrm{i} x_{k} c_{k}}=\exp \left[-\mathrm{i} \bar{x} \sum_{k=1}^{n} c_{k}\right] \exp \left[-\mathrm{i} \sum_{k=1}^{n-1}\left(x_{k}-x_{k+1}\right)\left(\sum_{j=1}^{k} c_{j}-\frac{k}{n} \sum_{j=1}^{k} c_{j}\right)\right] .
$$

Proof. With the definition of $\bar{x}$, we have

$$
\operatorname{det}\left(e^{\mathrm{i} x_{i} \lambda_{j}}\right)=e^{\mathrm{i} \bar{x} \sum_{k=1}^{n} \lambda_{k}} \operatorname{det}\left(e^{\mathrm{i}\left(x_{i}-\bar{x}\right) \lambda_{j}}\right) .
$$

Next we recall the Abel's identity as follows. In fact, we have

$$
\sum_{k=1}^{n} x_{k} y_{k}=y_{n} \sum_{k=1}^{n} x_{k}+\sum_{k=1}^{n-1}\left(y_{k}-y_{k+1}\right)\left(\sum_{j=1}^{k} x_{j}\right)
$$

Now we expand the determinant $\operatorname{det}\left(e^{\mathrm{i}\left(x_{i}-\bar{x}\right) \lambda_{j}}\right)$ as below:

$$
\operatorname{det}\left(e^{\mathrm{i}\left(x_{i}-\bar{x}\right) \lambda_{j}}\right)=\sum_{\sigma \in S_{n}} \operatorname{sign}(\sigma) \prod_{k=1}^{n} e^{\mathrm{i}\left(x_{k}-\bar{x}\right) \lambda_{\sigma(k)}}=\sum_{\sigma \in S_{n}} \operatorname{sign}(\sigma) \exp \left(\mathrm{i} \sum_{k=1}^{n}\left(x_{k}-\bar{x}\right) \lambda_{\sigma(k)}\right),
$$

where $\operatorname{sign}(\sigma)= \pm 1$ for odd $(-1)$ or even $(+1)$ permutation. By using Abel's identity, we have

$$
\begin{aligned}
\sum_{k=1}^{n} \lambda_{\sigma(k)}\left(x_{k}-\bar{x}\right) & =\left(x_{n}-\bar{x}\right) \sum_{k=1}^{n} \lambda_{\sigma(k)}+\sum_{k=1}^{n-1}\left(x_{k}-x_{k+1}\right)\left(\sum_{j=1}^{k} \lambda_{\sigma(j)}\right) \\
& =\sum_{k=1}^{n-1}\left(x_{k}-x_{k+1}\right)\left(\sum_{j=1}^{k} \lambda_{\sigma(j)}\right)-\left(\bar{x}-x_{n}\right) \sum_{j=1}^{n} \lambda_{j} .
\end{aligned}
$$

Again, by using Abel's identity, we have

$$
n \bar{x}=\sum_{k=1}^{n} 1 \cdot x_{k}=x_{n} \sum_{k=1}^{n} 1+\sum_{k=1}^{n-1}\left(x_{k}-x_{k+1}\right)\left(\sum_{j=1}^{k} 1\right)=n x_{n}+\sum_{k=1}^{n-1} k\left(x_{k}-x_{k+1}\right) .
$$

That is,

$$
\bar{x}-x_{n}=\sum_{k=1}^{n-1} \frac{k}{n}\left(x_{k}-x_{k+1}\right) .
$$


Finally, we have

$$
\begin{aligned}
\sum_{k=1}^{n} \lambda_{\sigma(k)}\left(x_{k}-\bar{x}\right) & =\sum_{k=1}^{n-1}\left(x_{k}-x_{k+1}\right)\left(\sum_{j=1}^{k} \lambda_{\sigma(j)}\right)-\left(\sum_{k=1}^{n-1} \frac{k}{n}\left(x_{k}-x_{k+1}\right)\right) \sum_{j=1}^{n} \lambda_{j} \\
& =\sum_{k=1}^{n-1}\left(x_{k}-x_{k+1}\right)\left(\sum_{j=1}^{k} \lambda_{\sigma(j)}-\frac{k}{n} \sum_{j=1}^{n} \lambda_{j}\right) .
\end{aligned}
$$

That is,

$$
\sum_{k=1}^{n} \lambda_{\sigma(k)}\left(x_{k}-\bar{x}\right)=\sum_{k=1}^{n-1}\left(x_{k}-x_{k+1}\right)\left(\sum_{j=1}^{k} \lambda_{\sigma(j)}-\frac{k}{n} \sum_{j=1}^{n} \lambda_{j}\right) .
$$

From the above discussion, we see that

$$
\operatorname{det}\left(e^{\mathrm{i} x_{i} \lambda_{j}}\right)=e^{\mathrm{i} \bar{x} \sum_{k=1}^{n} \lambda_{k}} \sum_{\sigma \in S_{n}} \operatorname{sign}(\sigma) \prod_{k=1}^{n-1} \exp \left[\mathrm{i}\left(x_{k}-x_{k+1}\right)\left(\sum_{j=1}^{k} \lambda_{\sigma(j)}-\frac{k}{n} \sum_{j=1}^{n} \lambda_{j}\right)\right] .
$$

Note that $\prod_{k=1}^{n} e^{-\mathrm{i} x_{k} c_{k}}=\exp \left[-\mathrm{i} \sum_{k=1}^{n} c_{k} x_{k}\right]$, where

$$
\sum_{k=1}^{n} c_{k} x_{k}=x_{n} \sum_{k=1}^{n} c_{k}+\sum_{k=1}^{n-1}\left(x_{k}-x_{k+1}\right)\left(\sum_{j=1}^{k} c_{j}\right) \text {. }
$$

Hence, via $x_{n}=\bar{x}-\sum_{k=1}^{n-1} \frac{k}{n}\left(x_{k}-x_{k+1}\right)$, it follows that

$$
\sum_{k=1}^{n} c_{k} x_{k}=\bar{x} \sum_{k=1}^{n} c_{k}+\sum_{k=1}^{n-1}\left(x_{k}-x_{k+1}\right)\left(\sum_{j=1}^{k} c_{j}-\frac{k}{n} \sum_{j=1}^{k} c_{j}\right),
$$

and we have the formula (2.6). This completes the proof.

In the following we derive a further simplified expression for the analytical formula in Theorem 2.1. By Theorem 2.1, we see that it suffices to calculate the following integral

$$
I\left(\boldsymbol{a}, \boldsymbol{b}: \boldsymbol{C}^{\text {diag }}\right):=\int_{\mathbb{R}^{n}}[\mathrm{~d} \boldsymbol{x}] \operatorname{det}\left(e^{\mathrm{i} x_{i} a_{j}}\right) \operatorname{det}\left(e^{\mathrm{i} x_{i} b_{j}}\right) \Delta(\boldsymbol{x})^{-2} \prod_{i=1}^{n} e^{-\mathrm{i} x_{i} C_{i i}} .
$$

Corollary 2.3. The joint pdf $q\left(\boldsymbol{C}^{\text {diag }} \mid \boldsymbol{a}, \boldsymbol{b}\right)$ of the diagonal part $\boldsymbol{C}^{\text {diag }}$ of the sum $\boldsymbol{C}=\boldsymbol{A}+\boldsymbol{B}$ is given by

$$
q\left(\boldsymbol{C}^{\text {diag }} \mid \boldsymbol{a}, \boldsymbol{b}\right)=\frac{M}{\Delta(\boldsymbol{a}) \Delta(\boldsymbol{b})} I\left(\boldsymbol{a}, \boldsymbol{b}: \boldsymbol{C}^{\text {diag }}\right),
$$

where $M$ is from (2.2) and

$$
I\left(\boldsymbol{a}, \boldsymbol{b}: \boldsymbol{C}^{\text {diag }}\right)=2 \pi \delta\left(\sum_{j=1}^{n}\left(a_{j}+b_{j}-\boldsymbol{C}_{j j}\right)\right) \sum_{\sigma, \tau \in S_{n}} \operatorname{sign}(\sigma \tau) \int_{\mathbb{R}^{n-1}} \frac{[\mathrm{d} \boldsymbol{u}]}{\widetilde{\Delta}(\boldsymbol{u})^{2}} \prod_{k=1}^{n-1} e^{\mathrm{i} u_{k} B_{k}(\sigma, \tau)}
$$

with

$$
B_{k}(\sigma, \tau):=\sum_{j=1}^{k}\left(a_{\sigma(j)}+b_{\tau(j)}-C_{j j}\right)-\frac{k}{n} \sum_{j=1}^{n}\left(a_{j}+b_{j}-C_{j j}\right) .
$$


Proof. Note that (2.9) is the reformulation of Theorem 2.1. So we focus on the expression $I(\boldsymbol{a}, \boldsymbol{b}$ : $\left.C^{\text {diag }}\right)$. From the formulae (2.5) and (2.6) we have

$$
\begin{aligned}
\operatorname{det}\left(e^{\mathrm{i} x_{i} a_{j}}\right) & =\exp \left[\mathrm{i} \bar{x} \sum_{k=1}^{n} a_{k}\right] \sum_{\sigma \in S_{n}} \operatorname{sign}(\sigma) \prod_{k=1}^{n-1} \exp \left[\mathrm{i}\left(x_{k}-x_{k+1}\right)\left(\sum_{j=1}^{k} a_{\sigma(j)}-\frac{k}{n} \sum_{j=1}^{n} a_{j}\right)\right], \\
\operatorname{det}\left(e^{\mathrm{i} x_{i} b_{j}}\right) & =\exp \left[\mathrm{i} \bar{x} \sum_{k=1}^{n} b_{k}\right] \sum_{\tau \in S_{n}} \operatorname{sign}(\tau) \prod_{k=1}^{n-1} \exp \left[\mathrm{i}\left(x_{k}-x_{k+1}\right)\left(\sum_{j=1}^{k} b_{\tau(j)}-\frac{k}{n} \sum_{j=1}^{n} b_{j}\right)\right] .
\end{aligned}
$$

and

$$
\prod_{k=1}^{n} e^{-\mathrm{i} x_{k} C_{k k}}=\exp \left[-\mathrm{i} \bar{x} \sum_{k=1}^{n} \boldsymbol{C}_{k k}\right] \exp \left[\mathrm{i} \sum_{k=1}^{n-1}\left(x_{k}-x_{k+1}\right)\left(-\sum_{j=1}^{k} \boldsymbol{C}_{j j}+\frac{k}{n} \sum_{j=1}^{k} \boldsymbol{C}_{j j}\right)\right] .
$$

And it follows that

$$
\begin{aligned}
& \operatorname{det}\left(e^{\mathrm{i} x_{i} a_{j}}\right) \operatorname{det}\left(e^{\mathrm{i} x_{i} b_{j}}\right) \prod_{k=1}^{n} e^{-\mathrm{i} x_{k} C_{k k}} \\
& =\exp \left[\mathrm{i} \bar{x} \sum_{j=1}^{n}\left(a_{j}+b_{j}-C_{j j}\right)\right] \sum_{\sigma, \tau \in S_{n}} \operatorname{sign}(\sigma \tau) \prod_{k=1}^{n-1} \exp \left[\mathrm{i}\left(x_{k}-x_{k+1}\right) B_{k}(\sigma, \tau)\right],
\end{aligned}
$$

where $B_{k}(\sigma, \tau)$ is defined in (2.11). We next perform the change of variables: $\left(x_{1}, \ldots, x_{n}\right) \rightarrow$ $\left(\bar{x}, u_{1}, \ldots, u_{n-1}\right)$, where $u_{k}=x_{k}-x_{k+1}$. The Jacobian of this transformation is given by

$$
J=\left|\frac{\partial\left(\bar{x}, u_{1}, \ldots, u_{n-1}\right)}{\partial\left(x_{1}, \ldots, x_{n}\right)}\right|=\left|\begin{array}{ccccc}
\frac{1}{n} & \frac{1}{n} & \cdots & \frac{1}{n} & \frac{1}{n} \\
1 & -1 & \cdots & 0 & 0 \\
0 & 1 & \ddots & 0 & 0 \\
\vdots & \vdots & \ddots & -1 & 0 \\
0 & 0 & \cdots & 1 & -1
\end{array}\right|=(-1)^{n-1}
$$

Then $\mathrm{d} x_{1} \wedge \cdots \wedge \mathrm{d} x_{n}=(-1)^{n-1} \mathrm{~d} \bar{x} \wedge \mathrm{d} u_{1} \wedge \cdots \wedge \mathrm{d} u_{n-1}$, that is, this transformation is volumepreserving, $[\mathrm{d} x]=\mathrm{d} \bar{x}[\mathrm{~d} u]$, where $[\mathrm{d} u]=\prod_{j=1}^{n-1} \mathrm{~d} u_{j}$. Now we have already known [13] that

$$
\delta(s)=\frac{1}{2 \pi} \int_{\mathbb{R}} e^{\mathrm{i} s t} \mathrm{~d} t
$$

This indicates that the first factor is given by

$$
\int_{\mathbb{R}} e^{\mathrm{i} \bar{x} \sum_{k=1}^{n}\left(a_{k}+b_{k}-c_{k}\right)} \mathrm{d} \bar{x}=2 \pi \delta\left(\sum_{k=1}^{n}\left(a_{k}+b_{k}-c_{k}\right)\right) .
$$

Finally, we have

$$
\begin{aligned}
I\left(\boldsymbol{a}, \boldsymbol{b}: \boldsymbol{C}^{\mathrm{diag}}\right) & =\int_{\mathbb{R}^{n}} \frac{[\mathrm{d} \boldsymbol{x}]}{\Delta(\boldsymbol{x})^{2}} \operatorname{det}\left(e^{\mathrm{i} x_{i} a_{j}}\right) \operatorname{det}\left(e^{\mathrm{i} x_{i} b_{j}}\right) \prod_{k=1}^{n} e^{-\mathrm{i} x_{k} C_{k k}} \\
& =\int_{\mathbb{R}} e^{\mathrm{i} \bar{x} \sum_{k=1}^{n}\left(a_{k}+b_{k}-c_{k}\right)} \mathrm{d} \bar{x} \sum_{\sigma, \tau \in S_{n}} \operatorname{sign}(\sigma \tau) \int_{\mathbb{R}^{n-1}} \frac{[\mathrm{d} \boldsymbol{u}]}{\widetilde{\Delta}(\boldsymbol{u})^{2}} \prod_{k=1}^{n-1} e^{\mathrm{i} u_{k} B_{k}(\sigma, \tau)} \\
& =2 \pi \delta\left(\sum_{j=1}^{n}\left(a_{j}+b_{j}-C_{j j}\right)\right) \sum_{\sigma, \tau \in S_{n}} \operatorname{sign}(\sigma \tau) \int_{\mathbb{R}^{n-1}} \frac{[\mathrm{d} \boldsymbol{u}]}{\widetilde{\Delta}(\boldsymbol{u})^{2}} \prod_{k=1}^{n-1} e^{\mathrm{i} u_{k} B_{k}(\sigma, \tau)},
\end{aligned}
$$


where

$$
\widetilde{\Delta}(\boldsymbol{u}):=\prod_{1 \leqslant i \leqslant j-1 \leqslant n-1}\left(u_{i}+u_{i+1}+\cdots+u_{j-1}\right) .
$$

This completes the proof.

Example 2.4. For the sum of two $2 \times 2$ random Hermitian matrices, we can derive concise expressions for the pdfs of diagonals of this sum. For $n=2$, for $\boldsymbol{a}=\left(a_{1}, a_{2}\right)$ with $a_{1} \geqslant a_{2}$ and $\boldsymbol{b}=\left(b_{1}, b_{2}\right)$ with $b_{1} \geqslant b_{2}$, the formula (2.10) can be simplifed as follows.

$$
I\left(\boldsymbol{a}, \boldsymbol{b}: \boldsymbol{C}^{\mathrm{diag}}\right)=2 \pi \delta\left(\sum_{j=1}^{2}\left(a_{j}+b_{j}-\boldsymbol{C}_{j j}\right)\right) \sum_{\sigma, \tau \in S_{2}} \operatorname{sign}(\sigma \tau) \int_{\mathbb{R}} \frac{\mathrm{d} u}{u^{2}} e^{\mathrm{i} u B(\sigma, \tau)},
$$

where

$$
B(\sigma, \tau):=a_{\sigma(1)}+b_{\tau(1)}-C_{11}-\frac{1}{2} \sum_{j=1}^{2}\left(a_{j}+b_{j}-C_{j j}\right) .
$$

Next, we calculate the integral:

$$
\int_{-\infty}^{+\infty} \frac{e^{\mathrm{i} u B(\sigma, \tau)}}{u^{2}} \mathrm{~d} u
$$

We apply the following formula for Fourier transform [13]

$$
\int_{-\infty}^{+\infty} \frac{e^{-\mathrm{i} w t}}{t^{n}} \mathrm{~d} t=-\mathrm{i} \pi \frac{(-\mathrm{i} w)^{n-1}}{\Gamma(n)} \operatorname{sgn}(w),
$$

where

$$
\operatorname{sgn}(w)= \begin{cases}1, & \text { if } w>0 \\ 0, & \text { if } w=0 \\ -1, & \text { if } w<0\end{cases}
$$

We can see that

$$
\int_{-\infty}^{+\infty} \frac{e^{-\mathrm{i} v t}}{t^{2}} \mathrm{~d} t=-\pi|v|
$$

and thus,

$$
\int_{-\infty}^{+\infty} \frac{e^{\mathrm{i} u B(\sigma)}}{u^{2}} \mathrm{~d} u=-\pi|B(\sigma, \tau)|
$$

From the above, we see that

$$
\sum_{\sigma, \tau \in S_{2}} \operatorname{sign}(\sigma \tau) \int_{\mathbb{R}} \frac{\mathrm{d} u}{u^{2}} e^{\mathrm{i} u B(\sigma, \tau)}=-\pi \sum_{\sigma, \tau \in S_{2}} \operatorname{sign}(\sigma \tau)|B(\sigma, \tau)| .
$$


Define $\alpha_{12}:=a_{1}-a_{2} \geqslant 0, \beta_{12}:=b_{1}-b_{2} \geqslant 0$, and $\tilde{\gamma}_{12}:=C_{11}-C_{22}$. Then (2.13) is rewritten as

$$
\begin{aligned}
I\left(\boldsymbol{a}, \boldsymbol{b}: \boldsymbol{C}^{\text {diag }}\right)= & 2 \pi \delta\left(\sum_{j=1}^{2}\left(a_{j}+b_{j}-\boldsymbol{C}_{j j}\right)\right) \\
& \times\left(-\frac{\pi}{2} \sum_{\sigma, \tau \in S_{2}} \operatorname{sign}(\sigma \tau)\left|\operatorname{sign}(\sigma) \alpha_{12}+\operatorname{sign}(\tau) \beta_{12}-\tilde{\gamma}_{12}\right|\right) .
\end{aligned}
$$

That is,

$$
\begin{aligned}
I\left(\boldsymbol{a}, \boldsymbol{b}: \boldsymbol{C}^{\text {diag }}\right)= & \pi^{2} \delta\left(\sum_{j=1}^{2}\left(a_{j}+b_{j}-\boldsymbol{C}_{j j}\right)\right)\left(\left|\alpha_{12}-\beta_{12}-\tilde{\gamma}_{12}\right|+\left|\alpha_{12}-\beta_{12}+\tilde{\gamma}_{12}\right|\right. \\
& \left.-\left|\alpha_{12}+\beta_{12}-\tilde{\gamma}_{12}\right|-\left|\alpha_{12}+\beta_{12}+\tilde{\gamma}_{12}\right|\right) .
\end{aligned}
$$

Therefore

$$
\begin{aligned}
q\left(\boldsymbol{C}^{\text {diag }} \mid \boldsymbol{a}, \boldsymbol{b}\right)= & \frac{1}{4 \alpha_{12} \beta_{12}} \delta\left(\sum_{j=1}^{2}\left(a_{j}+b_{j}-C_{j j}\right)\right)\left(\left|\alpha_{12}+\beta_{12}-\tilde{\gamma}_{12}\right|+\left|\alpha_{12}+\beta_{12}+\tilde{\gamma}_{12}\right|\right. \\
& \left.-\left|\alpha_{12}-\beta_{12}-\tilde{\gamma}_{12}\right|-\left|\alpha_{12}-\beta_{12}+\tilde{\gamma}_{12}\right|\right) .
\end{aligned}
$$

\section{The pdf of eigenvalues of the sum of random Hermitian matrices}

The derivative principle is formally put forward in [6]. The authors of [6] derived this result in the abstract level, i.e., in the regime of Lie algebra, and they used this result to obtain the distribution

of eigenvalues of random marginals of a multipartite random pure state. Later, Mejía, Zapata, and Botero rederived this result in Random Matrix Theory (RMT) [18], and they used this result to study the difference between two random mixed quantum states. The following version of the derivative principle is from [18].

Proposition 3.1 (The derivative principle). Let $\mathbf{Z}$ be an $n \times n$ random matrix drawn from a unitarily invariant random matrix ensemble, $p_{\mathbf{Z}}$ the joint eigenvalue distribution for $\mathbf{Z}$ and $q_{\mathbf{Z}}$ the joint distribution of the diagonal elements of $\mathbf{Z}$. Then

$$
p_{\mathbf{Z}}(\lambda)=\frac{1}{\prod_{k=1}^{n} k !}(-1)^{\left(\begin{array}{c}
n \\
2
\end{array}\right)} \Delta(\lambda) \Delta\left(\partial_{\lambda}\right) q_{\mathbf{Z}}(\lambda),
$$

where $\Delta(\lambda)=\prod_{i<j}\left(\lambda_{i}-\lambda_{j}\right)$ is the Vandermonde determinant and $\Delta\left(\partial_{\lambda}\right)$ the differential operator $\prod_{i<j}\left(\frac{\partial}{\partial_{\lambda_{i}}}-\frac{\partial}{\partial_{\lambda_{j}}}\right)$.

\subsection{A new derivation of the pdf by the derivative principle}

With the derivative principle, we can relate the pdf of the eigenvalues of the sum of two random Hermitian matrices with given spectra to the pdf of diagonals of this sum. 
In the following, we will use the derivative principle to rederive the pdf of eigenvalues of sum of two random Hermitian matrices with given spectra.

Theorem 3.2. Assume that two random Hermitian matrices $\boldsymbol{A}$ and $\boldsymbol{B}$ chosen uniformly on the unitary orbits $\mathcal{U}(\boldsymbol{a})$ and $\mathcal{U}(\boldsymbol{b})$, respectively, then the joint $p d f p(\boldsymbol{c} \mid \boldsymbol{a}, \boldsymbol{b})$ of the eigenvalues $\boldsymbol{c}$ of the sum $\boldsymbol{C}=\boldsymbol{A}+\boldsymbol{B}$ is given by derivative principle (3.1)

$$
p(\boldsymbol{c} \mid \boldsymbol{a}, \boldsymbol{b})=\frac{1}{\prod_{k=1}^{n} k !}(-1)^{\left(\begin{array}{c}
n \\
2
\end{array}\right)} \Delta(\boldsymbol{c}) \Delta\left(\partial_{c}\right) q(\boldsymbol{c} \mid \boldsymbol{a}, \boldsymbol{b}) .
$$

where $q(\boldsymbol{c} \mid \boldsymbol{a}, \boldsymbol{b})$ is from (2.9) by replacing $\boldsymbol{C}^{\text {diag }}$ as $\boldsymbol{c}$. Moreover,

$$
p(\boldsymbol{c} \mid \boldsymbol{a}, \boldsymbol{b})=\frac{\prod_{k=1}^{n} \Gamma(k)}{\left.(2 \pi)^{n} n ! \mathrm{i}^{n} \begin{array}{l}
n \\
2
\end{array}\right)} \frac{\Delta(\boldsymbol{c})}{\Delta(\boldsymbol{a}) \Delta(\boldsymbol{b})} \int_{\mathbb{R}^{n}} \frac{[\mathrm{d} \boldsymbol{x}]}{\Delta(\boldsymbol{x})} \operatorname{det}\left(e^{\mathrm{i} x_{i} a_{j}}\right) \operatorname{det}\left(e^{\mathrm{i} x_{i} b_{j}}\right) \prod_{k=1}^{n} e^{-\mathrm{i} x_{k} c_{k}} .
$$

Proof. Note that

$$
\left(\frac{\partial}{\partial_{c_{i}}}-\frac{\partial}{\partial_{c_{j}}}\right) q(\boldsymbol{c} \mid \boldsymbol{a}, \boldsymbol{b})=\frac{M}{\Delta(\boldsymbol{a}) \Delta(\boldsymbol{b})} \int \operatorname{det}\left(e^{\mathrm{i} x_{i} a_{j}}\right) \operatorname{det}\left(e^{\mathrm{i} x_{i} b_{j}}\right) \Delta(\boldsymbol{x})^{-2}\left(\frac{\partial}{\partial_{c_{i}}}-\frac{\partial}{\partial_{c_{j}}}\right) \prod_{k=1}^{n} e^{-\mathrm{i} x_{k} c_{k}} \mathrm{~d} x_{k},
$$

where

$$
\left(\frac{\partial}{\partial_{c_{i}}}-\frac{\partial}{\partial_{c_{j}}}\right) \prod_{k=1}^{n} e^{-i x_{k} c_{k}}=(-\mathrm{i})\left(x_{i}-x_{j}\right) \prod_{k=1}^{n} e^{-\mathrm{i} x_{k} c_{k}}
$$

Thus,

$$
\begin{aligned}
\Delta\left(\partial_{c}\right) \prod_{k=1}^{n} e^{-\mathrm{i} x_{k} c_{k}} & =\prod_{i<j}(-\mathrm{i})\left(x_{i}-x_{j}\right) \prod_{k=1}^{n} e^{-\mathrm{i} x_{k} c_{k}} \\
& =\frac{1}{\mathrm{i}\left(\begin{array}{c}
n \\
2
\end{array}\right)} \Delta(x) \prod_{k=1}^{n} e^{-\mathrm{i} x_{k} c_{k}} .
\end{aligned}
$$

Therefore,

$$
\Delta\left(\partial_{\boldsymbol{c}}\right) q(\boldsymbol{c} \mid \boldsymbol{a}, \boldsymbol{b})=\frac{1}{\left.\mathrm{i}^{(} \begin{array}{l}
n \\
2
\end{array}\right)} \frac{M}{\Delta(\boldsymbol{a}) \Delta(\boldsymbol{b})} \int \frac{[\mathrm{d} \boldsymbol{x}]}{\Delta(\boldsymbol{x})} \operatorname{det}\left(e^{\mathrm{i} x_{i} a_{j}}\right) \operatorname{det}\left(e^{\mathrm{i} x_{i} b_{j}}\right) \prod_{k=1}^{n} e^{-\mathrm{i} x_{k} c_{k}} .
$$

Substituting it into the right hand side of (3.1), we have

$$
\begin{aligned}
& \frac{1}{\prod_{k=1}^{n} k !}(-1)^{\left(\begin{array}{c}
n \\
2
\end{array}\right)} \Delta(\boldsymbol{c}) \Delta\left(\partial_{c}\right) q(\boldsymbol{c} \mid \boldsymbol{a}, \boldsymbol{b}) \\
& =\frac{(-1)^{\left(\begin{array}{c}
n \\
2
\end{array}\right)}}{n ! \prod_{k=1}^{n} \Gamma(k)} \frac{M}{\mathrm{i}^{\left(\begin{array}{c}
n \\
2
\end{array}\right)}} \frac{\Delta(\boldsymbol{c})}{\Delta(\boldsymbol{a}) \Delta(\boldsymbol{b})} \int \frac{[\mathrm{d} \boldsymbol{x}]}{\Delta(\boldsymbol{x})} \operatorname{det}\left(e^{\mathrm{i} x_{i} a_{j}}\right) \operatorname{det}\left(e^{\mathrm{i} x_{i} b_{j}}\right) \prod_{k=1}^{n} e^{-\mathrm{i} x_{k} c_{k}} \\
& =\frac{\prod_{k=1}^{n} \Gamma(k)}{\left.(2 \pi)^{n} n ! i^{(} \begin{array}{l}
n \\
2
\end{array}\right)} \frac{\Delta(\boldsymbol{c})}{\Delta(\boldsymbol{a}) \Delta(\boldsymbol{b})} \int \frac{[\mathrm{d} \boldsymbol{x}]}{\Delta(\boldsymbol{x})} \operatorname{det}\left(e^{\mathrm{i} x_{i} a_{j}}\right) \operatorname{det}\left(e^{\mathrm{i} x_{i} b_{j}}\right) \prod_{k=1}^{n} e^{-\mathrm{i} x_{k} c_{k}} .
\end{aligned}
$$

This is exactly the application of derivative principle, and it yields the joint eigenvalue distribution. 
Remark 3.3. Denote by $\lambda(\boldsymbol{X})$ the vector whose components consisting of eigenvalues in a nonincreasing order of Hermitian matrix $X$. Given two $2 \times 2$ Hermitian matrices $A$ and $B$ with $\lambda(\boldsymbol{A})=\left(a_{1}, a_{2}\right):=\boldsymbol{a}$ and $\lambda(\boldsymbol{B})=\left(b_{1}, b_{2}\right)=\boldsymbol{b}$. We also denote $\boldsymbol{C}=\boldsymbol{A}+\boldsymbol{B}$ and $\lambda(\boldsymbol{C})=\left(c_{1}, c_{2}\right):=$ c. Denote $I=\left(\left|\alpha_{12}-\beta_{12}\right|, \alpha_{12}+\beta_{12}\right)$, where $\alpha_{12}=a_{1}-a_{2} \geqslant 0$ and $\beta_{12}=b_{1}-b_{2} \geqslant 0$. The pdf of $C=U \widehat{a} U^{\dagger}+V \widehat{b} V^{\dagger}$ is given as [22]

$$
\begin{aligned}
p(\boldsymbol{c} \mid \boldsymbol{a}, \boldsymbol{b})= & \frac{c_{1}-c_{2}}{2\left(a_{1}-a_{2}\right)\left(b_{1}-b_{2}\right)}\left(1_{I}\left(c_{1}-c_{2}\right)-1_{-I}\left(c_{1}-c_{2}\right)\right) \\
& \times \delta\left(c_{1}+c_{2}-a_{1}-a_{2}-b_{1}-b_{2}\right),
\end{aligned}
$$

which can be also derived from (2.18) and the derivative principle.

\subsection{The pdf of eigenvalues of the sum of random Hermitian matrices from GUE ensemble}

Recall that the standard complex normal random variable or standard complex Gaussian random variable is a complex random variable $z$ whose real and imaginary parts are independent normally distributed random variables with mean zero and variance $\frac{1}{2}$. We use the notation $z \sim N_{C}(0,1)=N\left(0, \frac{1}{2}\right)+\sqrt{-1} N\left(0, \frac{1}{2}\right)$ to denote the fact that $z$ is the standard complex normal random variable.

The so-called $\operatorname{GUE}(n)$ ensemble is the class of complex Hermitian random matrices $A=$ $\left(a_{i j}\right)_{n \times n}$, generated in the following way:

$$
A=\frac{1}{2}\left(Z+Z^{\dagger}\right),
$$

where $\mathbf{Z}=\left(z_{i j}\right)_{n \times n}$ is the standard complex Gaussian random matrix, i.e., $z_{i j} \sim N_{\mathbb{C}}(0,1)$ are i.i.d. for all $i, j$. From this, we see that

$$
a_{i j}=\frac{1}{2}\left(z_{i j}+\bar{z}_{j i}\right)
$$

If $i=j$, then $a_{i i}=\operatorname{Re}\left(z_{i i}\right) \sim N\left(0, \frac{1}{2}\right)$; if $i \neq j$, then $a_{i j} \sim N_{\mathbb{C}}\left(0, \frac{1}{2}\right)$. Moreover, the density functions of $a_{i j}$ are given by

$$
p\left(a_{i j}\right)= \begin{cases}\frac{1}{\sqrt{\pi}} e^{-a_{i i}^{2},} & \text { if } i=j ; \\ p\left(a_{i j}\right)=\frac{2}{\pi} e^{-2\left|a_{i j}\right|^{2}}, & \text { if } i \neq j .\end{cases}
$$

Therefore the pdf of a random matrix $A \in \mathrm{GUE}(n)$ is given by the following:

$$
p(A)=\frac{2^{\left(\begin{array}{c}
n \\
2
\end{array}\right)}}{\pi^{\frac{n^{2}}{2}}} \exp \left(-\operatorname{Tr}\left(A^{2}\right)\right) .
$$

As already known [2],

$$
\int_{\mathbb{R}^{n}} \Delta(x)^{2} \exp (-\langle x, x\rangle)[\mathrm{d} x]=(2 \pi)^{\frac{n}{2}} 2^{-\frac{n^{2}}{2}} \prod_{k=1}^{n} k ! .
$$


The pdf of eigenvalues $\boldsymbol{a}=\left(a_{1}, a_{2}, \ldots, a_{n}\right)$ of $\boldsymbol{A}=\boldsymbol{U} \widehat{a} \boldsymbol{U}^{\dagger}$ is given by

$$
\frac{2^{\left(\begin{array}{c}
n \\
2
\end{array}\right)}}{\pi^{\frac{n}{2}} \prod_{k=1}^{n} k !} \Delta(\boldsymbol{a})^{2} \exp (-\langle\boldsymbol{a}, \boldsymbol{a}\rangle), \quad \boldsymbol{a} \in \mathbb{R}^{n} .
$$

Here $\langle\cdot, \cdot\rangle$ is the Euclid inner product.

Proposition 3.4. The pdf of $s=\left(s_{1}, \ldots, s_{n}\right)$ eigenvalues of the sum $S=\sum_{k=1}^{K} A_{k}$, where all $A_{k} \in$ $\operatorname{GUE}(n)$ are i.i.d., is given by

$$
p_{S, K}(s)=\frac{\left(\frac{2}{K}\right)^{\left(\begin{array}{c}
n \\
2
\end{array}\right)}}{(K \pi)^{\frac{n}{2}} \prod_{j=1}^{n} j !} \Delta(s)^{2} \exp \left(-\frac{1}{K}\langle s, s\rangle\right), \quad s \in \mathbb{R}^{n} .
$$

Proof. As an illustration, we show the corresponding result for $K=2$ and $S=A_{1}+A_{2}$. The proof for an arbitrary positive integer $K$ goes similarly. Firstly we work out the pdf of the diagonal part of $\boldsymbol{W}$. In fact,

$$
S^{\operatorname{diag}}=\operatorname{diag}\left(a_{11}^{(1)}+a_{11}^{(2)}, a_{22}^{(1)}+a_{22}^{(2)}, \ldots, a_{n n}^{(1)}+a_{n n}^{(2)}\right),
$$

where $a_{i i}^{(1)}, a_{j j}^{(2)} \sim N\left(0, \frac{1}{2}\right)$ are i.i.d. Gaussian random variables. Then the pdf $q\left(S^{\text {diag }}\right)$ of $S^{\text {diag }}$ is given

$$
q\left(S^{\text {diag }}\right)=\prod_{i=1}^{n} q\left(S_{i i}\right)
$$

where

$$
q\left(S_{i i}\right)=(\varphi \star \varphi)\left(S_{i i}\right)=\frac{1}{\sqrt{2 \pi}} \exp \left(-\frac{1}{2} S_{i i}^{2}\right)
$$

for $i=1, \cdots, n$. Here $\varphi(x)=\frac{1}{\sqrt{\pi}} e^{-x^{2}}$ and $\star$ means the convolution product. Thus

$$
q\left(S^{\text {diag }}\right)=\prod_{i=1}^{n} \frac{1}{\sqrt{2 \pi}} \exp \left(-\frac{1}{2} S_{i i}^{2}\right)=\left(\frac{1}{2 \pi}\right)^{\frac{n}{2}} \exp \left(-\frac{1}{2} \sum_{i=1}^{n} S_{i i}^{2}\right) .
$$

By using Proposition 3.1, we have

$$
\begin{aligned}
p_{S}(s) & =p_{A_{1}+A_{2}}(s)=\frac{1}{\prod_{k=1}^{n} k !}(-1)^{\left(\begin{array}{c}
n \\
2
\end{array}\right)} \Delta(s) \Delta\left(\partial_{s}\right) q(s) \\
& =\frac{1}{(2 \pi)^{\frac{n}{2}} \prod_{k=1}^{n} k !} \Delta(s)^{2} \exp \left(-\frac{1}{2}\langle s, s\rangle\right) .
\end{aligned}
$$

This completes the proof.

Note that this same result can be derived as a direct corollary of the (3.5) in the section. The above working amply demonstrate the consistency. 


\subsection{The connection with Golden-Thompson inequality}

Let $\mathrm{d} \mu(\boldsymbol{H})=p(\boldsymbol{H})[\mathrm{d} \boldsymbol{H}]$, where $p(\boldsymbol{H})$ the pdf which is given by (3.7). Denote

$$
\mathbb{E}_{\boldsymbol{H} \in \mathrm{GUE}(n)}[f(\boldsymbol{H})]=\int_{\mathrm{GUE}(n)} f(\boldsymbol{H}) \mathrm{d} \mu(\boldsymbol{H}),
$$

where $f(\boldsymbol{H})$ is the functional calculus of the function $f$. By the spectral decomposition of $\boldsymbol{H}$ : $\boldsymbol{H}=\boldsymbol{U} \Lambda \boldsymbol{U}^{\dagger}$, where $\Lambda=\operatorname{diag}\left(\lambda_{1}, \ldots, \lambda_{n}\right)$ with $+\infty>\lambda_{1}>\cdots>\lambda_{n}>-\infty$, and $\boldsymbol{U} \in \mathrm{U}(n) / \mathbb{T}$. Here $\mathbb{T}$ is the maximal torus of $\boldsymbol{U}(n)$, and for $f$ in (3.14) matrix valued $f(\boldsymbol{H})=\boldsymbol{U} f(\Lambda) \boldsymbol{U}^{\dagger}$, where $f(\Lambda)=\operatorname{diag}\left(f\left(\lambda_{1}\right), \ldots, f\left(\lambda_{n}\right)\right)$. Then we have [20]

$$
[\mathrm{d} \boldsymbol{H}]=\frac{\operatorname{vol}(\mathrm{U}(n))}{(2 \pi)^{n}} \Delta(\lambda)^{2}[\mathrm{~d} \Lambda] \mathrm{d} \nu(\boldsymbol{U}),
$$

where

$$
\operatorname{vol}(\mathrm{U}(n))=\frac{2^{n} \pi_{\left(\begin{array}{c}
n+1 \\
2
\end{array}\right)}^{n}}{\prod_{k=1}^{n} \Gamma(k)}
$$

is the volume associated with the Haar measure on $\mathrm{U}(n)$ and $\mathrm{d} v(\boldsymbol{U})$ is the normalized Haar measure over $\mathrm{U}(n)$ in the sense that

$$
\int_{\mathrm{U}(n)} \mathrm{d} v(\boldsymbol{U})=1
$$

We get that

$$
\mathrm{d} \mu(\boldsymbol{H})=\frac{2_{\left(\begin{array}{c}
n \\
2
\end{array}\right)}}{\pi^{\frac{n}{2}} \prod_{k=1}^{n} \Gamma(k)} \Delta(\lambda)^{2} \exp \left(-\operatorname{Tr}\left(\Lambda^{2}\right)\right)[\mathrm{d} \Lambda] \mathrm{d} \nu(\boldsymbol{U}) .
$$

Moreover,

$$
\begin{aligned}
\mathbb{E}_{\boldsymbol{H} \in \mathrm{GUE}(n)}[f(\boldsymbol{H})]= & \frac{2^{\left(\begin{array}{c}
n \\
2
\end{array}\right)}}{\pi^{\frac{n}{2}} \prod_{k=1}^{n} \Gamma(k)} \int_{+\infty>\lambda_{1}>\cdots>\lambda_{n}>-\infty} \Delta(\lambda)^{2} \exp \left(-\operatorname{Tr}\left(\Lambda^{2}\right)\right)[\mathrm{d} \Lambda] \\
& \times \int \mathrm{d} v(\boldsymbol{U}) \boldsymbol{U} f(\Lambda) \boldsymbol{U}^{+} \\
= & \kappa_{n}(f) \cdot \mathbb{1}_{n}
\end{aligned}
$$

where

$$
\begin{aligned}
\kappa_{n}(f) & \stackrel{\text { def }}{=} \frac{2^{\left(\begin{array}{c}
n \\
2
\end{array}\right)}}{n \pi^{\frac{n}{2}} \prod_{k=1}^{n} \Gamma(k)} \int_{+\infty>\lambda_{1}>\cdots>\lambda_{n}>-\infty} \operatorname{Tr}(f(\Lambda)) \Delta(\lambda)^{2} \exp \left(-\operatorname{Tr}\left(\Lambda^{2}\right)\right)[\mathrm{d} \Lambda] \\
& =\frac{1}{n} \frac{2^{\left(\begin{array}{c}
n \\
2
\end{array}\right)}}{\pi^{\frac{n}{2}} \prod_{k=1}^{n} k !} \int_{\mathbb{R}^{n}} \operatorname{Tr}(f(\Lambda)) \Delta(\lambda)^{2} \exp \left(-\operatorname{Tr}\left(\Lambda^{2}\right)\right)[\mathrm{d} \Lambda] \\
& =\frac{1}{n} \int_{\mathbb{R}^{n}} \operatorname{Tr}(f(\Lambda)) p(\Lambda)[\mathrm{d} \Lambda]
\end{aligned}
$$


and

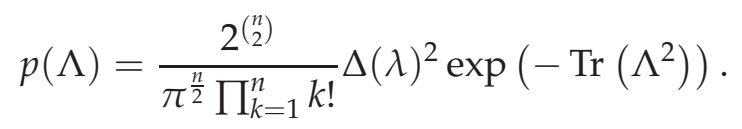

Note that in the above reasoning, we used the fact that

$$
\int_{\mathrm{U}(n)} \mathrm{d} v(\boldsymbol{U}) \boldsymbol{U} f(\Lambda) \boldsymbol{U}^{\dagger}=\frac{\operatorname{Tr}(f(\Lambda))}{n} \mathbb{1}_{n}
$$

Proposition 3.5 ([17]). Let

$$
H_{k}(x)=e^{x^{2}}\left(-\frac{\mathrm{d}}{\mathrm{d} x}\right)^{k} e^{-x^{2}}=k ! \sum_{i=0}^{\left[\frac{k}{2}\right]}(-1)^{i} \frac{(2 x)^{k-2 i}}{i !(k-2 i) !}, \quad k=0,1,2, \ldots
$$

be the $k$-th Hermite polynomial. Denote $\varphi_{k}(x)=\frac{1}{\sqrt{2^{k} k ! \sqrt{\pi}}} e^{-\frac{x^{2}}{2}} H_{k}(x)$. Then the pdf of a generic eigenvalue of a Hermitian random matrix from $\mathrm{GUE}(n)$ is given by

$$
p(x)=\frac{1}{n} \sum_{k=0}^{n-1} \varphi_{k}^{2}(x)=\varphi_{n}^{2}(x)-\sqrt{1+\frac{1}{n}} \varphi_{n-1}(x) \varphi_{n+1}(x), \quad x \in \mathbb{R} .
$$

In summary, we get that

$$
\mathbb{E}_{\boldsymbol{H} \in \mathrm{GUE}(n)}[f(\boldsymbol{H})]=\left(\int_{-\infty}^{\infty} f(x) p(x) \mathrm{d} x\right) \cdot \mathbb{1}_{n} .
$$

Here $p(x)$ is taken from (3.17).

Proposition 3.6 ([11]). It holds that

$$
\mathbb{E}_{\boldsymbol{H} \in \mathrm{GUE}(n)}\left[e^{t \boldsymbol{H}}\right]=\kappa_{n}(\exp , t) \cdot \mathbb{1}_{n}, \quad t \in \mathbb{R},
$$

where the constant $\kappa_{n}(\exp , t)$ is given by

$$
\kappa_{n}(\exp , t)=\int_{-\infty}^{\infty} e^{t x} p(x) \mathrm{d} x=e^{\frac{t^{2}}{4}} F\left(1-n, 2 ;-\frac{t^{2}}{2}\right),
$$

and $F$ is the confluent hypergeometric function defined by $F(a, c ; z) \stackrel{\text { def }}{=} \sum_{k=0}^{\infty} \frac{(a)_{k} z^{k}}{(c)_{k} k !}$ for $a, c, z \in \mathbb{C}$ such that $c \notin \mathbb{Z} \backslash \mathbb{N}$. Here $(a)_{k}:=\prod_{j=0}^{k-1}(a+j)$ is the Pochhammer notation.

Furthermore, based on Propositions 3.6 and 3.4, we can easily derive the following identity: Let $\boldsymbol{C} \stackrel{\text { def }}{=} \boldsymbol{A}+\boldsymbol{B}$ whose eigenvalues consist of the vector $\boldsymbol{c}=\left(c_{1}, \ldots, c_{n}\right)$. Then

$$
\begin{aligned}
\mathbb{E}_{\boldsymbol{A}, \boldsymbol{B} \in \mathrm{GUE}(n)}[f(\boldsymbol{A}+\boldsymbol{B})] & =\frac{1}{2^{\frac{n}{2}} \pi^{\frac{n^{2}}{2}}} \int f(\boldsymbol{C}) \exp \left(-\frac{1}{2} \operatorname{Tr}\left(\boldsymbol{C}^{2}\right)\right)[\mathrm{d} \boldsymbol{C}] \\
& =\kappa_{n} \cdot \mathbb{1}_{n}
\end{aligned}
$$

where

$$
\kappa_{n} \stackrel{\text { def }}{=} \frac{1}{n} \int_{\mathbb{R}^{n}} \operatorname{Tr}(f(\widehat{\boldsymbol{c}})) p_{C, 2}(\boldsymbol{c})[\mathrm{d} \boldsymbol{c}]
$$


It is well known that, for any two $n \times n$ Hermitian matrices $X$ and $Y$, the Golden-Thompson inequality holds:

$$
\operatorname{Tr}\left(e^{X+Y}\right) \leqslant \operatorname{Tr}\left(e^{X} e^{Y}\right)
$$

Define the ratio

$$
\alpha_{n} \stackrel{\text { def }}{=} \frac{\mathbb{E}_{X, Y \in \operatorname{GUE}(n)}\left[\operatorname{Tr}\left(e^{\boldsymbol{X}} e^{\boldsymbol{Y}}\right)\right]}{\mathbb{E}_{\boldsymbol{X}, \boldsymbol{Y} \in \operatorname{GUE}(n)}\left[\operatorname{Tr}\left(e^{\boldsymbol{X}+\boldsymbol{Y}}\right)\right]}
$$

Then

$$
\alpha_{n}=\frac{F\left(1-n, 2 ;-\frac{1}{2}\right)^{2}}{F(1-n, 2 ;-1)}
$$

For small numbers $n=2,3$ and 4 , we can analytically obtain that $\alpha_{2}=\frac{25}{24} \approx e^{0.040822}, \alpha_{3}=\frac{1369}{1248} \approx$ $e^{0.0925383}$ and $\alpha_{4}=\frac{130321}{112128} \approx e^{0.15036}$. In fact, we have a more interesting result, which seems that the leading asymptotic form is $e^{2(\sqrt{2}-1) \sqrt{n}}$ (see Theorem 3.7 with its proof). Thus, we observe that the ratio $\alpha_{n}$ exponentially increases with the matrix size $n$. This partly answers the question proposed by Forrester and Thompson in [9].

In what follows, we will derive the asymptotic form of $\alpha_{n}$. In order to do that, we need the explicit relationship between Laguerre polynomials and Kummer's functions (Kummer's function is also called confluent hypergeometric function $F(a, c ; z)$, mentioned in Proposition 3.6). Some details about the notions of Laguerre polynomails and Kummer's function and its relationship can be found in [10]. Now we can prove the following result.

Theorem 3.7. It holds that

$$
\lim _{n \rightarrow \infty} \frac{\ln \alpha_{n}}{\sqrt{n}}=2(\sqrt{2}-1)
$$

Proof. Using Eq.(13.6.19) in [10], we find the explicit relationship between Laguerre polynomials and Kummer's functions given as follows. For an arbitrary natural number $n>0, \alpha \in \mathbb{R}$, and $x \in \mathbb{R}$, we have

$$
F(-n, \alpha+1 ; x)=\frac{n !}{(\alpha+1)_{n}} L_{n}^{(\alpha)}(x)=\left(\begin{array}{c}
n+\alpha \\
n
\end{array}\right) L_{n}^{(\alpha)}(x),
$$

where $\left(\begin{array}{c}n+\alpha \\ n\end{array}\right)$ is the generalized binomial coefficient. Next, replacing $n$ by $n-1$ and letting $\alpha=1$ in Eq. (3.24), we get that

$$
F(1-n, 2 ; x)=\frac{1}{n} L_{n-1}^{(1)}(x) .
$$

The Laguerre polynomials' asymptotic behavior for large $n$, but fixed $\alpha$ and $x>0$, is given by [4]:

$$
L_{n}^{(\alpha)}(-x)=\frac{(n+1)^{\frac{\alpha}{2}-\frac{1}{4}}}{2 \sqrt{\pi}} \frac{e^{-\frac{x}{2}}}{x^{\frac{\alpha}{2}+\frac{1}{4}}} e^{2 \sqrt{(n+1) x}}\left(1+O\left(\frac{1}{\sqrt{n+1}}\right)\right)
$$


From Eq. (3.25), we infer that, for large $n$ and fixed $x>0$,

$$
F(1-n, 2 ;-x)=\frac{1}{2 \sqrt{\pi}} \frac{\exp \left(2 \sqrt{n x}-\frac{x}{2}\right)}{(n x)^{\frac{3}{4}}}\left(1+O\left(\frac{1}{\sqrt{n}}\right)\right)
$$

Based on the observation, substituting $x=\frac{1}{2}, 1$, respectively, in Eq. (3.26) and plugging them into the expression of $\alpha_{n}$, we derive that

$$
\alpha_{n} \sim \sqrt{\frac{2}{\pi}} n^{-\frac{3}{4}} e^{2(\sqrt{2}-1) \sqrt{n}}
$$

for large $n$. Therefore,

$$
\lim _{n \rightarrow \infty} \frac{\ln \alpha_{n}}{\sqrt{n}}=2(\sqrt{2}-1)
$$

This competes the proof.

\section{Applications in quantum information theory}

Recall that a positive semi-definite matrix of unit trace are called a density matrix which are used to describe a state of quantum systems. Denote the set of all density matrices of size $n$ by $\mathrm{D}\left(\mathbb{C}^{n}\right)$. Let $\boldsymbol{A}=\frac{1}{2} \rho_{1}$ and $\boldsymbol{B}=\frac{1}{2} \rho_{2}$, where $\rho_{1}, \rho_{2} \in \mathrm{D}\left(\mathbb{C}^{2}\right)$, the set of density matrices of all qubit states. Then $\alpha=\frac{1}{2}(1-\mu, \mu), \beta=\frac{1}{2}(1-v, v)$ and $\gamma=(1-\lambda, \lambda)$ where $\mu, v \in(0,1 / 2)$ and $\lambda \in(0,1)$. Thus

$$
p(\lambda \mid \mu, v)= \begin{cases}\frac{\frac{1}{2}-\lambda}{\left(\frac{1}{2}-\mu\right)\left(\frac{1}{2}-v\right)}, & \text { if } \lambda \in\left[T_{0}, T_{1}\right] \\ \frac{\lambda-\frac{1}{2}}{\left(\frac{1}{2}-\mu\right)\left(\frac{1}{2}-v\right)}, & \text { if } \lambda \in\left[1-T_{1}, 1-T_{0}\right]\end{cases}
$$

where $T_{0}:=T_{0}(\mu, v)=\frac{\mu+v}{2}$ and $T_{1}:=T_{1}(\mu, v)=\frac{1-|\mu-v|}{2}$. The normalization of $p(\lambda \mid \mu, v)$ is easily obtained by using mathematical software. We omit it here.

Let $C^{\text {diag }}=\operatorname{diag}(1-x, x)$ for $x \in[0,1]$. Then $C^{\text {diag }}=A^{\text {diag }}+B^{\text {diag }}$ since $C=A+B$. Thus there exist $t, s \in[0,1]$ such that

$$
\begin{aligned}
1-x & =(1-t) \frac{1-\mu}{2}+t \frac{\mu}{2}+(1-s) \frac{1-v}{2}+s \frac{v}{2} \\
x & =t \frac{1-\mu}{2}+(1-t) \frac{\mu}{2}+s \frac{1-v}{2}+(1-s) \frac{v}{2} .
\end{aligned}
$$

This implies that

$$
1-2 x=\left(\frac{1}{2}-\mu\right)(1-2 t)+\left(\frac{1}{2}-v\right)(1-2 s) .
$$

For fixed $\mu, v \in\left[0, \frac{1}{2}\right]$, we see that as a function of arguments $(t, s) \in[0,1] \times[0,1]$,

$$
\left(\frac{1}{2}-\mu\right)(1-2 t)+\left(\frac{1}{2}-v\right)(1-2 s)
$$


has the maximum $1-\mu-v$ and the minimum $\mu+v-1$. Therefore,

$$
\mu+v-1 \leqslant 1-2 x \leqslant 1-\mu-v \Longleftrightarrow x \in\left[T_{0}, 1-T_{0}\right] .
$$

We also have that

$$
q(x \mid \mu, v)=\frac{1}{2\left(\frac{1}{2}-\mu\right)\left(\frac{1}{2}-v\right)}\left(\left|x-T_{0}\right|+\left|x-\left(1-T_{0}\right)\right|-\left|x-T_{1}\right|-\left|x-\left(1-T_{1}\right)\right|\right) .
$$

Furthermore, simplifying it into the following form:

$$
q(x \mid \mu, v)=\frac{1}{\left(\frac{1}{2}-\mu\right)\left(\frac{1}{2}-v\right)} \begin{cases}x-T_{0}, & \text { if } x \in\left[T_{0}, T_{1}\right] \\ T_{1}-T_{0}, & \text { if } x \in\left[T_{1}, 1-T_{1}\right] \\ -x+\left(1-T_{0}\right), & \text { if } x \in\left[1-T_{1}, 1-T_{0}\right] .\end{cases}
$$

Note that $q(x \mid \mu, v)$ is concentrated on $\left[T_{0}, 1-T_{0}\right] \subset[0,1]$ and $q(x \mid \mu, v)$ is vanished on $[0,1] \backslash\left[T_{0}, 1-\right.$ $T_{0}$. The normalization can be checked directly as follows:

$$
\begin{aligned}
\int_{0}^{1} q(x \mid \mu, v) \mathrm{d} x= & \frac{1}{\left(\frac{1}{2}-\mu\right)\left(\frac{1}{2}-v\right)} \\
& \times\left[\int_{T_{0}}^{T_{1}}\left(x-T_{0}\right) \mathrm{d} x+\int_{T_{1}}^{1-T_{1}}\left(T_{1}-T_{0}\right) \mathrm{d} x+\int_{1-T_{1}}^{1-T_{0}}\left(-x+\left(1-T_{0}\right)\right) \mathrm{d} x\right] \\
= & \frac{\left[\frac{1}{2} x^{2}-T_{0} x\right]_{T_{0}}^{T_{1}}+\left(T_{1}-T_{0}\right)\left(1-2 T_{1}\right)+\left[-\frac{1}{2} x^{2}+\left(1-T_{0}\right) x\right]_{1-T_{1}}^{1-T_{0}}}{\left(\frac{1}{2}-\mu\right)\left(\frac{1}{2}-v\right)}
\end{aligned}
$$

implying that

$$
\int_{0}^{1} q(x \mid \mu, v) \mathrm{d} x=\frac{\left(T_{1}-T_{0}\right)\left(1-T_{1}-T_{0}\right)}{\left(\frac{1}{2}-\mu\right)\left(\frac{1}{2}-v\right)}=1
$$

\subsection{Uniform average distance between two orbits}

We consider the following quantum Jensen-Shannon divergence which is defined by

$$
\operatorname{QJSD}\left(\rho_{1}, \rho_{2}\right):=\frac{1}{2}\left[\mathrm{~S}\left(\rho_{1}|| \bar{\rho}\right)+\mathrm{S}\left(\rho_{2}|| \bar{\rho}\right)\right],
$$

where $\bar{\rho}=\frac{1}{2} \rho_{1}+\frac{1}{2} \rho_{2}$ and $S\left(\rho_{i}|| \bar{\rho}\right)=\operatorname{Tr}\left(\rho_{i}\left(\ln \rho_{i}-\ln \bar{\rho}\right)\right)$ is the so-called relative entropy. Clearly, $\operatorname{QJSD}\left(\rho_{1}, \rho_{2}\right)=\mathrm{S}\left(\frac{1}{2} \rho_{1}+\frac{1}{2} \rho_{2}\right)-\frac{1}{2} S\left(\rho_{1}\right)-\frac{1}{2} S\left(\rho_{2}\right)$. We may use our results obtained previously to investigate the average QJSD between two unitary orbits:

$$
\begin{aligned}
& \iint \mathrm{d} \mu_{\text {Haar }}(U) \mathrm{d} \mu_{\text {Haar }}(V) \operatorname{QJSD}\left(U \rho_{1} U^{\dagger}, V \rho_{2} V^{\dagger}\right) \\
& =\iint \mathrm{d} \mu_{\text {Haar }}(U) \mathrm{d} \mu_{\text {Haar }}(V) S\left(\frac{1}{2} U \rho_{1} U^{\dagger}+\frac{1}{2} V \rho_{2} V^{\dagger}\right)-\frac{1}{2} \mathrm{H}_{2}(\mu)-\frac{1}{2} \mathrm{H}_{2}(v) \\
& =\int_{T_{0}}^{T_{1}} \mathrm{H}_{2}(\lambda) \frac{\frac{1}{2}-\lambda}{\left(\frac{1}{2}-\mu\right)\left(\frac{1}{2}-v\right)} \mathrm{d} \lambda+\int_{1-T_{1}}^{1-T_{0}} \mathrm{H}_{2}(\lambda) \frac{\lambda-\frac{1}{2}}{\left(\frac{1}{2}-\mu\right)\left(\frac{1}{2}-v\right)} \mathrm{d} \lambda-\frac{1}{2} \mathrm{H}_{2}(\mu)-\frac{1}{2} \mathrm{H}_{2}(v),
\end{aligned}
$$


where $\mathrm{H}_{2}(x):=-x \ln x-(1-x) \ln (1-x)$ is the binary entropy function for $x \in[0,1]$.

This gives an explicit expression about the uniform average QJSD, i.e., uniform average quantum Jensen-Shannon divergence, between two distinctive isospectral quantum states. Define

$$
\overline{\operatorname{QJSD}}\left(\mathcal{O}_{\mu}, \mathcal{O}_{v}\right):=\iint \mathrm{d} \mu_{\text {Haar }}(U) \mathrm{d} \mu_{\text {Haar }}(V) \operatorname{QJSD}\left(U \rho_{1} U^{\dagger}, V \rho_{2} V^{\dagger}\right),
$$

where $\mathcal{O}_{x}=\left\{U \operatorname{diag}(1-x, x) U^{+}: U \in \mathrm{U}(2)\right\}$ for $x \in[0,1]$. Thus we see that

$$
\overline{\operatorname{QJSD}}\left(\mathcal{O}_{\mu}, \mathcal{O}_{v}\right)=\int_{0}^{1} \mathrm{H}_{2}(\lambda) p(\lambda \mid \mu, v) \mathrm{d} \lambda-\frac{1}{2} \mathrm{H}_{2}(\mu)-\frac{1}{2} \mathrm{H}_{2}(v),
$$

where

$$
\begin{aligned}
\int_{0}^{1} \mathrm{H}_{2}(\lambda) p(\lambda \mid \mu, v) \mathrm{d} \lambda= & \frac{1}{\left(\frac{1}{2}-\mu\right)\left(\frac{1}{2}-v\right)} \\
& \times\left[\int_{T_{0}}^{T_{1}} \mathrm{H}_{2}(\lambda)\left(\frac{1}{2}-\lambda\right) \mathrm{d} \lambda+\int_{1-T_{1}}^{1-T_{0}} \mathrm{H}_{2}(\lambda)\left(\lambda-\frac{1}{2}\right) \mathrm{d} \lambda\right] .
\end{aligned}
$$

Note that by using Mathematica 10.0, we get that

$$
\begin{aligned}
F_{0}(x) & :=\int \mathrm{H}_{2}(x) \mathrm{d} x=\frac{1}{4}(1-x)^{2}[2 \ln (1-x)-1]-\frac{1}{4} x^{2}(2 \ln x-1) \\
F_{1}(x) & :=\int \mathrm{H}_{2}(x) x \mathrm{~d} x \\
& =\frac{1}{4}(1-x)^{2}[2 \ln (1-x)-1]-\frac{1}{9}(1-x)^{3}[3 \ln (1-x)-1]-\frac{1}{9} x^{3}(3 \ln x-1) .
\end{aligned}
$$

We also find that, for $x \in[0,1]$

$$
\begin{aligned}
& F_{0}(1-x)+F_{0}(x)=0 \\
& F_{1}(x)-F_{1}(1-x)=F_{0}(x)
\end{aligned}
$$

Thus,

$$
\overline{\operatorname{QJSD}}\left(\mathcal{O}_{\mu}, \mathcal{O}_{v}\right)=\frac{\left[\frac{1}{2} F_{0}(\lambda)-F_{1}(\lambda)\right]_{T_{0}}^{T_{1}}+\left[F_{1}(\lambda)-\frac{1}{2} F_{0}(\lambda)\right]_{1-T_{1}}^{1-T_{0}}}{\left(\frac{1}{2}-\mu\right)\left(\frac{1}{2}-v\right)}-\frac{1}{2} \mathrm{H}_{2}(\mu)-\frac{1}{2} \mathrm{H}_{2}(v) .
$$

Denote $\overline{\operatorname{QJSD}}\left(\mathcal{O}_{\mu}, \mathcal{O}_{v}\right)$ as $\varphi(\mu, v)$, where $(\mu, v) \in\left[0, \frac{1}{2}\right] \times\left[0, \frac{1}{2}\right]$. That is,

$$
\varphi(\mu, v):=\frac{\Phi(\mu, v)}{\left(\frac{1}{2}-\mu\right)\left(\frac{1}{2}-v\right)}-\frac{1}{2} \mathrm{H}_{2}(\mu)-\frac{1}{2} \mathrm{H}_{2}(v),
$$

where

$$
\begin{aligned}
\Phi(\mu, v)= & {\left[\frac{1}{2} F_{0}(\lambda)-F_{1}(\lambda)\right]_{T_{0}}^{T_{1}}+\left[F_{1}(\lambda)-\frac{1}{2} F_{0}(\lambda)\right]_{1-T_{1}}^{1-T_{0}} } \\
= & \frac{1}{2}\left(F_{0}\left(T_{1}\right)+F_{0}\left(1-T_{1}\right)-F_{0}\left(T_{0}\right)-F_{0}\left(1-T_{0}\right)\right) \\
& +\left(F_{1}\left(T_{0}\right)+F_{1}\left(1-T_{0}\right)-F_{1}\left(T_{1}\right)-F_{1}\left(1-T_{1}\right)\right)
\end{aligned}
$$


and $(\mu, v) \in\left[0, \frac{1}{2}\right] \times\left[0, \frac{1}{2}\right]$. Thus using (4.7), we get

$$
\Phi(\mu, v)=F_{1}\left(T_{0}\right)+F_{1}\left(1-T_{0}\right)-F_{1}\left(T_{1}\right)-F_{1}\left(1-T_{1}\right) .
$$

And therefore we have

$$
\varphi(\mu, v)=\frac{F_{1}\left(T_{0}\right)+F_{1}\left(1-T_{0}\right)-F_{1}\left(T_{1}\right)-F_{1}\left(1-T_{1}\right)}{\left(\frac{1}{2}-\mu\right)\left(\frac{1}{2}-v\right)}-\frac{1}{2} \mathrm{H}_{2}(\mu)-\frac{1}{2} \mathrm{H}_{2}(v) .
$$

The symmetry of $\mu$ and $v$ is obvious. The maximum is achieved at $(0,0)$. That is, $\varphi(0,0)=$ $\frac{1}{3} \ln 2+\frac{1}{6}$. And the minimum is $\varphi\left(\frac{1}{2}, \frac{1}{2}\right)=0$. The case where $\mu$ or $v=0$ corresponds to the pure state, and the case where $\mu$ or $v=\frac{1}{2}$ corresponds to the maximum mixed state. Hence, the quantum Jensen-Shannon divergence is the largest in the case of two pure states, and smallest in the case of two maximally mixed states. In addition, $\varphi\left(\frac{1}{2}, 0\right)=\varphi\left(0, \frac{1}{2}\right)=-\frac{3}{4} \ln \frac{3}{4}=0.2158$.

\subsection{Average coherence of uniform mixture of two orbits}

In this subsection, we will see that the derivative principle is naturally related to quantum coherence of relative entropy, a hot topic which is intensive studied from various viewpoints [3]. Let us recall that the coherence of relative entropy proposed by Baumgratz et al is quantified by the relative entropy between a given state and the nearest incoherent state to the original one. Specifically, it is given by $\mathscr{C}(\rho)=\mathrm{S}\left(\rho_{\text {diag }}\right)-\mathrm{S}(\rho)$. Surprisedly, it is determined by both the diagonal part and its eigenvalues of this given state. When we study the quantum coherence of relative entropy of a random quantum state and its typicality, the derivative principle can be naturally applied. Recently, indeed, we considered the average coherence and its typicality [19, 21]. Here we will present more subtle result about quantum coherence of a random state limited to the qubit case.

In the following we consider the average coherence of uniform mixture of two unitary orbits with respective prescribed spectra, i.e.,

$$
\begin{aligned}
& \bar{C}\left(\mathcal{O}_{\mu}, \mathcal{O}_{v}\right):=\iint \mathrm{d} \mu_{\text {Haar }}(U) \mathrm{d} \mu_{\text {Haar }}(V) \mathscr{C}\left(\frac{1}{2} U \rho_{1} U^{+}+\frac{1}{2} V \rho_{2} V^{+}\right) \\
& =\iint \mathrm{d} \mu_{\text {Haar }}(U) \mathrm{d} \mu_{\text {Haar }}(V)\left[\mathrm{S}\left(\left(\frac{1}{2} U \rho_{1} U^{\dagger}+\frac{1}{2} V \rho_{2} V^{\dagger}\right)_{\text {diag }}\right)-\mathrm{S}\left(\frac{1}{2} U \rho_{1} U^{\dagger}+\frac{1}{2} V \rho_{2} V^{\dagger}\right)\right] \\
& =\int_{0}^{1} \mathrm{H}_{2}(x) q(x \mid \mu, v) \mathrm{d} x-\int_{0}^{1} \mathrm{H}_{2}(\lambda) p(\lambda \mid \mu, v) \mathrm{d} \lambda
\end{aligned}
$$

where

$$
\int_{0}^{1} \mathrm{H}_{2}(x) q(x \mid \mu, v) \mathrm{d} x=\frac{\left[F_{1}(x)-T_{0} F_{0}(x)\right]_{T_{0}}^{T_{1}}+\left(T_{1}-T_{0}\right)\left[F_{0}(x)\right]_{T_{1}}^{1-T_{1}}+\left[\left(1-T_{0}\right) F_{0}(x)-F_{1}(x)\right]_{1-T_{1}}^{1-T_{0}}}{\left(\frac{1}{2}-\mu\right)\left(\frac{1}{2}-v\right)} .
$$


Denote $\overline{\mathscr{C}}\left(\mathcal{O}_{\mu}, \mathcal{O}_{v}\right)$ as $\psi(\mu, v)$, where $(\mu, v) \in\left[0, \frac{1}{2}\right] \times\left[0, \frac{1}{2}\right]$. That is,

$$
\psi(\mu, v):=\frac{\Psi(\mu, v)-\Phi(\mu, v)}{\left(\frac{1}{2}-\mu\right)\left(\frac{1}{2}-v\right)}
$$

where

$$
\begin{aligned}
\Psi(\mu, v) & :=\left[F_{1}(x)-T_{0} F_{0}(x)\right]_{T_{0}}^{T_{1}}+\left(T_{1}-T_{0}\right)\left[F_{0}(x)\right]_{T_{1}}^{1-T_{1}}+\left[\left(1-T_{0}\right) F_{0}(x)-F_{1}(x)\right]_{1-T_{1}}^{1-T_{0}} \\
& =T_{0} \cdot F_{0}\left(T_{0}\right)+\left(1-T_{0}\right) \cdot F_{0}\left(1-T_{0}\right)-T_{1} \cdot F_{0}\left(T_{1}\right)-\left(1-T_{1}\right) \cdot F_{0}\left(1-T_{1}\right)-\Phi(\mu, v)
\end{aligned}
$$

and $\Phi(\mu, v)$ is from Eq. (4.11) and $(\mu, v) \in\left[0, \frac{1}{2}\right] \times\left[0, \frac{1}{2}\right]$. Thus using (4.7) again, we get

$$
\Psi(\mu, v)+\Phi(\mu, v)=\left(2 T_{0}-1\right) \cdot F_{0}\left(T_{0}\right)-\left(2 T_{1}-1\right) \cdot F_{0}\left(T_{1}\right)
$$

This implies that

$$
\psi(\mu, v):=\frac{\left(2 T_{0}-1\right) F_{0}\left(T_{0}\right)-\left(2 T_{1}-1\right) F_{0}\left(T_{1}\right)-2 \Phi(\mu, v)}{\left(\frac{1}{2}-\mu\right)\left(\frac{1}{2}-v\right)},
$$

We can see that $\psi(\mu, v)$ is completely determined by two integrals $\int_{0}^{1} \mathrm{H}_{2}(x) q(x \mid \mu, v) \mathrm{d} x$ and $\int_{0}^{1} \mathrm{H}_{2}(x) p(x \mid \mu, v) \mathrm{d} x$ in (4.13). The biggest difference between the two integrals appears at $(0,0)$, while the difference approaches zero at $\left(\frac{1}{2}, \frac{1}{2}\right)$. The substraction of the two integrals yields the function $\psi(\mu, v)$. The symmetry of $\psi$ on the parameters $\mu$ and $v$ can be easily checked. The maximum is achieved at $(0,0)$. Specifically we have $\psi(0,0)=\frac{2}{3}(1-\ln 2)=0.2046$. The minimum is the limit at $\left(\frac{1}{2}, \frac{1}{2}\right)$, i.e., $\psi\left(\frac{1}{2}, \frac{1}{2}\right)=0$. In addition, $\psi\left(0, \frac{1}{2}\right)=\psi\left(\frac{1}{2}, 0\right)=\frac{1}{2}-\frac{3}{8} \ln 3=0.0880$.

\section{Concluding remarks}

In this paper, we investigate a variant of Horn's problem, i.e., we identify the pdf of the diagonals of the sum of two random Hermitian matrices with given spectra. We then use it, together with the derivative principle, to re-derive the pdf of the eigenvalues of the sum of two random Hermitian matrices with given spectra. Zuber's recent results on the same problem can be also recovered. We then apply these results further to derive the analytical expressions of eigenvalues of the sum of two random Hermitian matrices from $\operatorname{GUE}(n)$ or Wishart ensemble by derivative principle, respectively. We also investigate the statistics of exponential of random matrices and connect them with Golden-Thompson inequality, and partially answer a question proposed by Forrester. The results obtained are also employed to analyze quantum Jensen-Shannon divergence between two unitary orbits with their prescribed spectra and quantum coherence of mixture of two unitary orbits. Although these applications in quantum information theory are just around 2-dimensional space, we believe our methods used in the present work can be extended to higher dimensional space. 


\section{Acknowledgement}

The authors sincerely thank the editor and the anonymous referees for their comments, especially the proposal for Theorem 3.7, which led to great improvements of this paper. The authors also thank Lin Huang for pointing out the connection with Laguerre polynomial. Lin Zhang is supported by Zhejiang Provincial Natural Science Foundation of China under grant no. LY17A010027 and also by National Natural Science Foundation of China (Nos.11971140, 11701259, 61771174). Hua Xiang is supported by National Natural Science Foundation of China (No.11571265) and NSFC-RGC (China-Hong Kong, No.11661161017).

\section{Appendices}

\section{A Zuber's derivation of the pdf}

Theorem A.1 (J-B. Zuber, [22]). Assume that two random matrices $A$ and B chosen uniformly on the unitary orbits $\mathcal{U}(\boldsymbol{a})$ and $\mathcal{U}(\boldsymbol{b})$, respectively, then the joint $p d f p(\boldsymbol{c} \mid \boldsymbol{a}, \boldsymbol{b})$ of the eigenvalues $\boldsymbol{c}$ of the sum $C:=A+B$ is given by the following integral

$$
p(\boldsymbol{c} \mid \boldsymbol{a}, \boldsymbol{b})=\text { const. } \Delta(\boldsymbol{c})^{2} \int_{\mathbb{R}^{n}}[\mathrm{~d} \boldsymbol{x}] \Delta(\boldsymbol{x})^{2} \mathcal{I}(\boldsymbol{a}, \mathrm{i} \boldsymbol{x}) \mathcal{I}(\boldsymbol{b}, \mathrm{i} \boldsymbol{x}) \overline{\mathcal{I}(\boldsymbol{c}, \mathrm{i} \boldsymbol{x})},
$$

where

$$
\mathcal{I}(\boldsymbol{a}, \boldsymbol{b})=\int_{\mathrm{U}(n)} \exp \left(\operatorname{Tr}\left(\widehat{\boldsymbol{a}} \boldsymbol{U} \widehat{\boldsymbol{b}} \boldsymbol{U}^{\dagger}\right)\right) \mathrm{d} \mu_{\mathrm{Haar}}(\boldsymbol{U})
$$

is the famous Harish-Chandra integral for which the explicit formula can be written down [14]:

$$
\mathcal{I}(\boldsymbol{a}, \boldsymbol{b})=\left(\prod_{k=1}^{n} \Gamma(k)\right) \frac{\operatorname{det}\left(e^{a_{i} b_{j}}\right)}{\Delta(\boldsymbol{a}) \Delta(\boldsymbol{b})} .
$$

Proposition A.2. The pdf of eigenvalues $\boldsymbol{c}$, given $\boldsymbol{a}$ and $\boldsymbol{b}$, is given by

$$
\begin{aligned}
p(\boldsymbol{c} \mid \boldsymbol{a}, \boldsymbol{b}) & =\frac{\prod_{k=1}^{n} \Gamma(k)}{\left.(2 \pi)^{n}(n !)^{2} \mathbf{i}_{2}^{n}\right)} \frac{\Delta(\boldsymbol{c})}{\Delta(\boldsymbol{a}) \Delta(\boldsymbol{b})} \int \frac{[\mathrm{d} \boldsymbol{x}]}{\Delta(\boldsymbol{x})} \operatorname{det}\left(e^{\mathrm{i} x_{i} a_{j}}\right) \operatorname{det}\left(e^{\mathrm{i} x_{i} b_{j}}\right) \operatorname{det}\left(e^{-\mathrm{i} x_{i} c_{j}}\right) \\
& =\frac{\prod_{k=1}^{n} \Gamma(k)}{\left.(2 \pi)^{n} n ! \mathbf{i}_{2}^{n}\right)} \frac{\Delta(\boldsymbol{c})}{\Delta(\mathbf{a}) \Delta(\mathbf{b})} \int \frac{[\mathrm{d} \boldsymbol{x}]}{\Delta(\boldsymbol{x})} \operatorname{det}\left(e^{\mathrm{i} x_{i} a_{j}}\right) \operatorname{det}\left(e^{\mathrm{i} x_{i} b_{j}}\right) \prod_{k=1}^{n} e^{-\mathrm{i} x_{k} c_{k}} .
\end{aligned}
$$

Proof. Note that we can use permutational symmetry of the integrand to replace $\operatorname{det}\left(e^{-\mathrm{i} x_{i} c_{j}}\right)$ by $n ! e^{-\mathrm{i}\langle x, c\rangle}=n ! \prod_{k=1}^{n} e^{-\mathrm{i} x_{k} c_{k}}$.

Note that (A.5) is exactly the same as (3.3), which is derived from $q(\boldsymbol{c} \mid \boldsymbol{a}, \boldsymbol{b})$ and the derivative principle in Section 3. 
Corollary A.3. The pdf of eigenvalues $\boldsymbol{c}$, given $\boldsymbol{a}$ and $\boldsymbol{b}$, is given by

$$
p(\boldsymbol{c} \mid \boldsymbol{a}, \boldsymbol{b})=\frac{\prod_{k=1}^{n} \Gamma(k)}{(2 \pi)^{n-1} n ! \mathbf{i}^{\left(\begin{array}{c}
n \\
2
\end{array}\right)}} \delta\left(\sum_{k=1}^{n}\left(a_{k}+b_{k}-c_{k}\right)\right) \frac{\Delta(\boldsymbol{c})}{\Delta(\boldsymbol{a}) \Delta(\boldsymbol{b})} J(\boldsymbol{a}, \boldsymbol{b}: \boldsymbol{c}),
$$

where $J(\boldsymbol{a}, \boldsymbol{b}: \boldsymbol{c})$ is given by

$$
J(\boldsymbol{a}, \boldsymbol{b}: \boldsymbol{c})=\sum_{\sigma, \tau \in S_{n}} \operatorname{sign}(\sigma \tau) \int_{\mathbb{R}^{n-1}} \frac{[\mathrm{d} \boldsymbol{u}]}{\widetilde{\Delta}(\boldsymbol{u})} \prod_{k=1}^{n-1} \exp \left[\mathrm{i} u_{k} A_{k}(\sigma, \tau)\right],
$$

and where

$$
A_{k}(\sigma, \tau)=\sum_{j=1}^{k}\left(a_{\sigma(j)}+b_{\tau(j)}-c_{j}\right)-\frac{k}{n} \sum_{j=1}^{n}\left(a_{j}+b_{j}-c_{j}\right) .
$$

Although an approach toward the integral in Theorem A.1 is sketched by Zuber in [22], we choose to reconstruct the details for reader's convenience. Note that there are a few different tricks from the way sketched by Zuber.

Proof. We simplify the two determinants in the integral of (A.5). Then applying (2.5), we get

$$
\begin{aligned}
\operatorname{det}\left(e^{\mathrm{i} x_{i} a_{j}}\right) \operatorname{det}\left(e^{\mathrm{i} x_{i} b_{j}}\right)= & e^{\mathrm{i} \bar{x} \sum_{k=1}^{n}\left(a_{k}+b_{k}\right)} \sum_{\sigma, \tau \in S_{n}} \operatorname{sign}(\sigma \tau) \\
& \times \prod_{k=1}^{n-1} \exp \left[\mathrm{i}\left(x_{k}-x_{k+1}\right)\left(\sum_{j=1}^{k}\left(a_{\sigma(j)}+b_{\tau(j)}\right)\right)-\frac{k}{n} \sum_{j=1}^{n}\left(a_{j}+b_{j}\right)\right] .
\end{aligned}
$$

We switch to the last term in the integral of (A.5). Thus, by using (2.7)

$$
\prod_{k=1}^{n} e^{-\mathrm{i} x_{k} c_{k}}=\exp \left[-\mathrm{i} \bar{x} \sum_{k=1}^{n} c_{k}\right] \exp \left[-\mathrm{i} \sum_{k=1}^{n-1}\left(x_{k}-x_{k+1}\right)\left(\sum_{j=1}^{k} c_{j}-\frac{k}{n} \sum_{j=1}^{k} c_{j}\right)\right] .
$$

Therefore, we have

$$
\begin{aligned}
& \operatorname{det}\left(e^{\mathrm{i} x_{i} a_{j}}\right) \operatorname{det}\left(e^{\mathrm{i} x_{i} b_{j}}\right) \prod_{k=1}^{n} e^{-\mathrm{i} x_{k} c_{k}} \\
& =e^{\mathrm{i} \bar{x} \sum_{k=1}^{n}\left(a_{k}+b_{k}-c_{k}\right)} \sum_{\sigma, \tau \in S_{n}} \operatorname{sign}(\sigma \tau) \prod_{k=1}^{n-1} \exp \left[\mathrm{i}\left(x_{k}-x_{k+1}\right) A_{k}(\sigma, \tau)\right] .
\end{aligned}
$$

where

$$
A_{k}(\sigma, \tau)=\sum_{j=1}^{k}\left(a_{\sigma(j)}+b_{\tau(j)}-c_{j}\right)-\frac{k}{n} \sum_{j=1}^{n}\left(a_{j}+b_{j}-c_{j}\right)
$$

It follows that

$$
\begin{aligned}
& \int_{\mathbb{R}^{n}} \frac{[\mathrm{d} x]}{\Delta(\boldsymbol{x})} \operatorname{det}\left(e^{\mathrm{i} x_{i} a_{j}}\right) \operatorname{det}\left(e^{\mathrm{i} x_{i} b_{j}}\right) \prod_{k=1}^{n} e^{-\mathrm{i} x_{k} c_{k}} \\
& =\int_{\mathbb{R}} e^{\mathrm{i} \bar{x} \sum_{k=1}^{n}\left(a_{k}+b_{k}-c_{k}\right)} \mathrm{d} \bar{x} \sum_{\sigma, \tau \in S_{n}} \operatorname{sign}(\sigma \tau) \int_{\mathbb{R}^{n-1}} \frac{[\mathrm{d} \boldsymbol{u}]}{\widetilde{\Delta}(\boldsymbol{u})} \prod_{k=1}^{n-1} \exp \left[\mathrm{i} u_{k} A_{k}(\sigma, \tau)\right],
\end{aligned}
$$


where

$$
\widetilde{\Delta}(\boldsymbol{u}):=\prod_{1 \leqslant i \leqslant j-1 \leqslant n-1}\left(u_{i}+u_{i+1}+\cdots+u_{j-1}\right) .
$$

Therefore, we see that

$$
\begin{aligned}
& \int_{\mathbb{R}^{n}} \frac{[\mathrm{d} x]}{\Delta(x)} \operatorname{det}\left(e^{\mathrm{i} x_{i} a_{j}}\right) \operatorname{det}\left(e^{\mathrm{i} x_{i} b_{j}}\right) \prod_{k=1}^{n} e^{-\mathrm{i} x_{k} c_{k}} \\
& =2 \pi \delta\left(\sum_{k=1}^{n}\left(a_{k}+b_{k}-c_{k}\right)\right) \sum_{\sigma, \tau \in S_{n}} \operatorname{sign}(\sigma \tau) \int_{\mathbb{R}^{n-1}} \frac{[\mathrm{d} \boldsymbol{u}]}{\widetilde{\Delta}(u)} \prod_{k=1}^{n-1} \exp \left[\mathrm{i} u_{k} A_{k}(\sigma, \tau)\right] .
\end{aligned}
$$

We are done.

\section{References}

[1] A. Alekseev, M. Podkopaeva, A. Szenes, A symplectic proof of the Horn inequalities, Adv. Math. 318, 711-736 (2017).

[2] G.E. Andrews, R. Askey, R. Roy, Special Functions, Cambridge University Press, 1999.

[3] T. Baumgratz, M. Cramer, M.B. Plenio, Quantifying Coherence, Phys. Rev. Lett. 113, 140401 (2014).

[4] D. Borwein, J.M. Borwein, and R.E. Crandall, Effective Laguerre Asymptotics, SIAM J. Numer. Anal. 46(6), 3285-3312 (2008).

[5] L. Cao, New Formulation and Uniqueness of Solutions to A. Horn's Problem, PhD Thesis, Drexel University (2012).

[6] M. Christandl, B. Doran, S. Kousidis, M. Walter, Eigenvalue distributions of reduced density matrices, Comm. Math. Phys. 332, 1-52 (2014).

[7] J. Faraut, Horn's problem and Fourier analysis, Tunisian J. Math. 1(4), 585-606 (2019).

[8] P.J. Forrester and J. Zhang, Co-rank 1 projections and the randomised Horn problem, arXiv:1905.05314

[9] P.J. Forrester and C.J. Thompson, The Golden-Thompson inequality: Historical aspects and random matrix applications, J. Math. Phys. 55, 023503 (2014).

[10] F.W.J. Olver, D.W. Lozier, R.F. Boisvert, and C.W. Clark, NIST Handbook of Mathematical Functions, Cambridge University Press (2010).

[11] U. Haagerup, S. Thorbijörnsen, Random matrices with complex Gaussian entries, Expo. Math. 21, 293-337 (2003). 
[12] A. Horn, Eigenvalues of sums of Hermitian matrices, Pacific J. Math. 12, 225-241 (1962).

[13] R.F. Hoskins, Delta Functions: An Introduction to Generalised Functions, 2nd Edition 2009, Woodhead Publishing Limited, Oxford (2011).

[14] C. Itzykson, J.B. Züber, The planar approximation. II, J. Math. Phys. 21, 411-421 (1980).

[15] A. Knutson, The symplectic and algebraic geometry of Horn's problem, Lin. Alg. App. 319, 61-81 (2000)

[16] S. Kumar, Eigenvalue statistics for the sum of two complex Wishart matrices, Europhys. Lett. 107, 60002 (2014).

[17] M.L. Mehta, Random Matrices, 3rd Edition, Elsevier Academic Press (2004).

[18] J. Mejía, C. Zapata, A. Botero, The difference between two random mixed quantum states: exact and asymptotic spectral analysis, J. Phys. A : Math. Theor. 50, 025301 (2017).

[19] L. Zhang, Average coherence and its typicality for random mixed quantum states, J. Phys. A : Math. Theor. 50, 155303 (2017).

[20] L. Zhang, S. Hong, Volume of the set of locally diagonable bipartite states, J. Phys. A : Math. Theor. 51, 385302 (2018).

[21] L. Zhang, U. Singh, A.K. Pati, Average subentropy, coherence and entanglement of random mixed quantum states, Ann. Phys. 377, 125-146 (2017).

[22] J.B. Zuber, Horn's problem and Harish-Chandra's integrals I. Probability density functions, Ann. Inst. Henri Poincaré, Comb. Phys. Interact. 5, 309-338 (2018). arXiv: 1705.01186v2 